

\title{
The system of representations of the Weil-Deligne group associated to an abelian variety
}

\author{
Rutger Noot
}

\begin{abstract}
Fix a number field $F \subset \mathbb{C}$, an abelian variety $A / F$ and let $G_{A}$ be the Mumford-Tate group of $A_{/ \mathbb{C}}$. After replacing $F$ by finite extension one can assume that, for every prime number $\ell$, the action of the absolute Galois group $\Gamma_{F}=\operatorname{Gal}(\bar{F} / F)$ on the étale cohomology group $\mathrm{H}_{\mathrm{t}}^{1}\left(A_{\bar{F}}, \mathbb{Q}_{\ell}\right)$ factors through a morphism $\rho_{\ell}: \Gamma_{F} \rightarrow G_{A}\left(\mathbb{Q}_{\ell}\right)$. Let $v$ be a valuation of $F$ and write $\Gamma_{F_{v}}$ for the absolute Galois group of the completion $F_{v}$. For every $\ell$ with $v(\ell)=0$, the restriction of $\rho_{\ell}$ to $\Gamma_{F_{v}}$ defines a representation ${ }^{\prime} W_{F_{v}} \rightarrow G_{A / \mathbb{Q}_{l}}$ of the WeilDeligne group.

It is conjectured that, for every $\ell$, this representation of ' $W_{F_{v}}$ is defined over $\mathbb{Q}$ as a representation with values in $G_{A}$ and that the system above, for variable $\ell$, forms a compatible system of representations of ' $W_{F_{v}}$ with values in $G_{A}$. A somewhat weaker version of this conjecture is proved for the valuations of $F$, where $A$ has semistable reduction and for which $\rho_{\ell}\left(\mathrm{Fr}_{v}\right)$ is neat.
\end{abstract}

\section{Introduction}

Let $F_{v}$ be a finite extension of the field $\mathbb{Q}_{p}$ (for some prime number $p$ ) and let $X$ be a proper and smooth variety over $F_{v}$. The Galois group $\Gamma_{F_{v}}=\operatorname{Gal}\left(\bar{F}_{v} / F_{v}\right)$ acts on the étale cohomology groups $\mathrm{H}^{i}\left(X_{\bar{F}_{v}}, \mathbb{Q}_{\ell}\right)$ for each prime number $\ell$ and each $i$. It is a major problem in arithmetic geometry to determine to what extent the properties of these representations are independent of $\ell$. To obtain such independence results, one has to consider the restrictions of the representations above to the Weil group $W_{F_{v}}$ of $F_{v}$. This is the subgroup formed by the elements of $\Gamma_{F_{v}}$, which induce an integral power of the Frobenius automorphism on the residue field of $F_{v}$.

In what follows it will always be assumed that $\ell \neq p$; the case where $\ell=p$ will be analysed in a later paper. Let us first assume that $X$ has good reduction at $v$, that is, that $X$ extends to a proper and smooth scheme over the ring of integers of $F_{v}$. This assumption implies that the inertia subgroup of $\Gamma_{F_{v}}$ acts trivially on the

MSC2000: primary 11G10; secondary 14K15, 14F20.

Keywords: abelian variety, compatible system of Galois representations, Weil-Deligne group. 
étale cohomology groups of $X$. Moreover, it follows from Deligne's work [1974a] on the Weil conjectures that the character of the representation of $W_{F_{v}}$ on each $\mathrm{H}^{i}\left(X_{\bar{F}_{v}}, \mathbb{Q}_{\ell}\right)$ has values in $\mathbb{Q}$ and is independent of $\ell \neq p$. In view of the triviality of the action of inertia, this amounts to a statement on the action of the subgroup of $\Gamma_{F_{v}}$ generated by a Frobenius element. We will summarise this statement by saying that the representations of $W_{F_{v}}$ on the $\mathrm{H}^{i}\left(X_{\bar{F}_{v}}, \mathbb{Q}_{\ell}\right)$ (for fixed $i$ and variable $\ell$ ) are defined over $\mathbb{Q}$ and that they form a compatible system of representations of $W_{F_{v}}$.

If $X$ only has potentially good reduction, the inertia group no longer acts trivially but its action on a given étale cohomology group of $X$ factors through a finite quotient. It is still conjectured that the system of $\mathrm{H}^{i}\left(X_{\bar{F}_{v}}, \mathbb{Q}_{\ell}\right)$ (as always for fixed $i$ and variable $\ell$ ) forms a compatible system of representations of $W_{F_{v}}$ which are defined over $\mathbb{Q}$, see for example [Serre 1994, 12.13?].

Serre actually states his conjecture in more generality because it applies to motives instead of varieties. The category of (pure) motives can be seen as an intermediate between varieties and their cohomology. It is a $\mathbb{Q}$-linear tannakian category, that is, an abelian category in which the morphisms between two objects form a $\mathbb{Q}$-vector space and which is equipped with "tensor products". All reasonable cohomology functors on the category of varieties factor through the category of motives so that any motive has cohomology groups in the same way as varieties do. We also refer to the cohomology groups of a motive $M$ as the realisations of $M$. There are different constructions of motives, depending on how the morphisms are defined, but in any case the category of motives has more morphisms and hence also more objects than the category of varieties.

In this paper we will consider the category of motives for absolute Hodge cycles developed in [Deligne and Milne 1982] where the morphisms are defined by absolute Hodge classes. This theory is particularly efficient for dealing with abelian varieties because the motivic Galois group of an abelian variety $A$ coincides with its Mumford-Tate group, defined by the Hodge structure on $\mathrm{H}_{\mathrm{B}}^{1}\left(A_{/ \mathbb{C}}, \mathbb{Q}\right)$.

The motivic version of the conjecture can be stated in terms of motivic Galois groups in the following way. In any good category of motives it is possible to associate a motivic Galois group to any subcategory. This group is a linear proalgebraic group over $\mathbb{Q}$. Its defining property is the fact that any category of motives is equivalent to the category of representations of its motivic Galois group. The motivic Galois group $G_{M}$ of any object $M$ is defined as the group associated to the tannakian category generated by $M$ and the Tate motive. The group $G_{M}$ is related to the étale cohomology of $M$ by the fact that, for each prime number $\ell$, the $\ell$-adic Galois representation associated to $M$ factors through $G_{M}\left(\mathbb{Q}_{\ell}\right)$. This implies that the corresponding representations of the Weil group factor through $G_{M / \mathbb{Q}_{\ell}}$. Serre's $\ell$-independence conjecture for objects in the $\otimes$-category generated by $M$ is then equivalent to the statement that the representations $W_{F_{v}} \rightarrow G_{M / \mathbb{Q}_{\ell}}$ 
of the Weil group form a compatible system defined over $\mathbb{Q}$, with values in $G_{M}$; see 2.3 for the precise definition.

For abelian varieties of CM type, the conjecture follows from the theory of complex multiplication developed by Shimura and Taniyama [1961], which yields a considerably more precise result. Indeed, let $F \subset \mathbb{C}$ be a number field, $A / F$ an abelian variety of $\mathrm{CM}$ type and $G_{A}$ the motivic Galois group of $A$. Serre [1968] constructs a commutative algebraic group $S_{F, \mathfrak{m}}$ and a canonical system of representations $\varphi_{\ell}: \Gamma_{F} \rightarrow S_{F, \mathfrak{m}}\left(\mathbb{Q}_{\ell}\right)$. By the theory of complex multiplication, the system of $\ell$-adic representations associated to $A$ is the image of the system $\left(\varphi_{\ell}\right)$ by a $\mathbb{Q}$-rational morphism $S_{F, \mathfrak{m}} \rightarrow G_{A}$. Moreover, Deligne [1982] gives an explicit description of the motivic Galois group of the category of abelian varieties potentially of CM type in terms of the Taniyama group.

More generally, if $F \subset \mathbb{C}$ is a number field, $A / F$ an abelian variety and $v$ a valuation of $F$ where $A$ has good reduction, the $\ell$-independence conjecture was proved in [Noot 2009], by a method and under additional hypotheses similar to those of the present paper. The case where $A$ has ordinary reduction at $v$ has been treated in [Noot 1995] by a completely different method. In the latter case, a stronger statement can be proved and it turns out that there is an element in $G_{A}(\mathbb{Q})$ that is conjugate to the $\ell$-adic image of Frobenius for all $\ell \neq p$. As noted in the introduction of [Noot 1995], this is not the case in general. The reader may consult the introduction to [Noot 2009] for a more detailed discussion.

One may ask if these results can be generalised without any assumptions on the reduction of $X$. Before discussing the properties of the Galois representations provided by the étale cohomology groups of a variety $X$, consider any $\ell$-adic representation $\rho_{\ell}$ of $\Gamma_{F_{v}}$ for $\ell \neq p$. By a theorem of Grothendieck (see [Serre and Tate 1968, Appendix; Deligne 1973, §2, §8]), the action of a sufficiently small open subgroup of the inertia group can be described using a single endomorphism $N_{\ell}$, the monodromy operator. The restriction of $\rho_{\ell}$ to the Weil group $W_{F_{v}}$ can then be encoded by giving $N_{\ell}$ together with a representation $\rho_{\ell}^{\prime}$ of $W_{F_{v}}$, which is trivial on an open subgroup of the inertia group. We will refer to such a pair $\left(\rho_{\ell}^{\prime}, N_{\ell}\right)$ as a representation of the Weil-Deligne group ${ }^{\prime} W_{F_{v}}$ of $F_{v}$. Where the $p$-adic étale cohomology is concerned, Fontaine's theory associates a representation of the Weil-Deligne group to any semistable $p$-adic representation as well, but we will not go into the details here.

It is conjectured that, applying the construction above to the system of Galois representations provided by the $\mathrm{H}^{i}\left(X_{\bar{F}_{v}}, \mathbb{Q}_{\ell}\right)$, for fixed $i$ and variable $\ell$, one obtains a compatible system of representations of ${ }^{\prime} W_{F_{v}}$ which are defined over $\mathbb{Q}$; see for example [Fontaine 1994, 2.4.3, conjecture $C_{W D}$ ] for a statement encompassing the $p$-adic representation. The conjecture on the $\ell$-independence of the representation of the Weil-Deligne group hinges on the monodromy-weight conjecture; see 
[Illusie 1994, §3]. This elusive conjecture is somewhat more accessible under the hypothesis that $X$ has semistable reduction, a hypothesis which implies in particular that the representations $\rho_{\ell}^{\prime}$ are trivial on the inertia subgroup of $W_{F_{v}}$. The action of inertia on $\mathrm{H}^{i}\left(X_{\bar{F}_{v}}, \mathbb{Q}_{\ell}\right)$ is then determined by the monodromy operator $N_{\ell}$. Even if $X$ has semistable reduction however, the monodromy-weight conjecture has so far only been proved under far more restrictive hypotheses. Apart from the cases where $X$ is a curve or an abelian variety, the main achievement is due to [Rapoport and Zink 1982], which treats the case where $X$ has dimension 2. We refer to [Ochiai 1999; Ito 2004] and the work of Scholze for some recent and very recent progress on the problems. It should finally be pointed out that the discussion above has an analogue in equal characteristics, which has proved much more accessible; see for example [Deligne 1980].

This paper aims to study the motivic version of Fontaine's $C_{W D}$ conjecture. Under some additional hypotheses, described below, we will prove the compatibility conjecture for the system ' $W_{F_{v}} \rightarrow G_{A / \mathbb{Q}_{\ell}}$ associated to an abelian variety $A$ defined over a number field $F \subset \mathbb{C}$. Here $v$ is a fixed valuation of $F$ and $\ell$ runs through the set of primes with $v(\ell)=0$.

The hypotheses are twofold. First of all, we need to assume that the number field $F$ is sufficiently big. We do not only need to ensure that $A$ has semistable reduction, but also that the Mumford-Tate group $G_{A}$ is connected and even that the Frobenius element at the given place of $F$ is weakly neat; see Definition 3.5. Secondly, in certain cases we will need to work in a group that is slightly larger than the Mumford-Tate group; see Section 3.3. The Mumford-Tate group coincides with the identity component of this larger group. Enlarging the group obviously weakens the notion of conjugacy. The precise result is Theorem 3.6 and all definitions used in the statement are given in Section 3.

The strategy of the proof is inspired by the previous paper [Noot 2009], which treats the good reduction case. The idea is first to prove the statement for tractable abelian varieties (called accomodantes [ibid.]). In Section 4, we recall the notion of a tractable abelian variety as well as some related constructions stemming from [ibid.]. Tractable abelian varieties have many endomorphisms and the theorem is proved by combining more or less classical results concerning abelian varieties and 1-motives with the, equally classical, work of Springer and Steinberg on conjugacy classes in linear algebraic groups. The necessary results on representations of the Weil-Deligne group associated to a 1-motive follow from the theory sketched in [Fontaine 1994] by adding the action of an endomorphism algebra throughout. We make extensive use of [Raynaud 1994], which allows the reduction to the case of strict 1-motives. This is discussed, together with the relevant prerequisites, in Sections 1 and 2. Using the results of these sections, proof of the main theorem for a tractable abelian variety is given in Sections 5 and 6. 
Once we have proved the main theorem for tractable abelian varieties, the general case can be deduced using the theory, developed in [Noot 2006], of lifting Galois representations along isogenies between the Mumford-Tate groups. This final step of the proof is carried out in Section 7. In order to construct the liftings of the Galois representations, one needs to extend the base field. At first, this leads to a proof of the main theorem over an uncontrollable extension of the base field. The results of Sections 1 and 2 are used again to deduce the theorem over the original base field. The condition that the Frobenius element is weakly neat is essential in this step.

In his thesis, Laskar [2011] generalises the results of this paper, as well as those of [Noot 2009], to a larger class of varieties. He proves the main theorem of [ibid.] for the absolute Hodge motive of any variety $X$ with good reduction belonging to the tannakian category generated by the motives of abelian varieties. Under somewhat more restrictive conditions, Laskar also generalises the results of the present paper, treating the case of curves, $K 3$ surfaces and a Fermat hypersurfaces with semistable reduction.

Another direction for generalisation is the case of 1-motives. In this context, an analogue of the theory of Mumford-Tate liftings remains to be developed. The analogue of our results in the case where the base field is a function field in characteristic $p$ seems inaccessible with the techniques used in this paper. Indeed, it would be necessary to develop a substitute for the theory of absolute Hodge motives and, most importantly, the concept of Mumford-Tate liftings.

\section{1-motives with $L$-action}

In this section, we indicate how the theory of 1-motives developed in [Raynaud 1994; Deligne 1974b, §10] works out for 1-motives with a given endomorphism field. Most of the statements are easy generalisations of those given in [Fontaine 1994] for the case where $L=\mathbb{Q}$. The results of this section will be applied to the study of the monodromy of an abelian variety with a given endomorphism algebra. Where these preliminary results are concerned, very little additional effort is required to deal with the more general case of 1-motives.

We also review the construction of the Weil-Deligne group of a local field and recall how to associate a representation of the Weil-Deligne group to a local Galois representation.

1.1. Generalities on 1-motives. In all of this section, $F$ is a finite extension of $\mathbb{Q}_{p}$ and $\ell, \ell^{\prime}$ are prime numbers.

Let $M$ be a 1-motive over $F$. By definition $M$ is a complex $u: Y \rightarrow G$ where $Y$ is a $K$-group scheme which is locally isomorphic, in the étale topology, to $\mathbb{Z}^{r}$ and $G$ is a semiabelian variety over $K$. In this complex, $Y$ is placed in degree -1 and 
$G$ in degree 0 . The semiabelian variety $G$ is an extension of an abelian variety $A$ of dimension $g$ by a torus $T$ of dimension $r^{\star}$. Let $d=d(M)=r+r^{\star}+2 g$.

One defines the $\ell$-adic realisations of $M$ following [Deligne 1974b, 10.1], taking projective limits. This differs by a trivial manipulation from Raynaud's definition [1994, 3.1] where an inductive limit is used. To be precise, for an integer $n$, we define $\operatorname{Tors}_{n}(M)$ as the $\mathrm{H}^{-1}$ of the complex

$$
\begin{aligned}
Y & \rightarrow Y \oplus G(\bar{F}) \rightarrow G(\bar{F}), \\
x & \mapsto(-n x,-u(x)), \\
& (x, y) \mapsto u(x)-n y,
\end{aligned}
$$

situated in degrees $-2,-1,0$. Here and in what follows, $\bar{F}$ is an algebraic closure of $F$. For any $\ell$ we put

$$
T_{\ell}(M)=\lim _{\longleftarrow} \operatorname{Tors}_{\ell^{n}}(M) \quad \text { and } \quad V_{\ell}(M)=T_{\ell}(M) \otimes_{\mathbb{Z}_{\ell}} \mathbb{Q}_{\ell} .
$$

Thus, $T_{\ell}(M)$ is a free $\mathbb{Z}_{\ell}$-module of rank $d$ and $V_{\ell}(M)$ is a $\mathbb{Q}_{\ell}$-vector space of dimension $d$. For each $\ell$, the absolute Galois group $\Gamma_{F}=\operatorname{Aut}_{F}(\bar{F})=\operatorname{Gal}(\bar{F} / F)$ acts naturally on $V_{\ell}$.

We fix a number field $L \subset \operatorname{End}^{0}(M)=\operatorname{End}(M) \otimes_{\mathbb{Z}} \mathbb{Q}$. The endomorphism ring $\operatorname{End}(M)$ can be interpreted either as the ring of endomorphisms of the complex $Y \rightarrow G$ or as the ring of endomorphisms of its image in the derived category $D^{b}$ (fppf); see [Raynaud 1994, 2.3]. The first interpretation of $\operatorname{End}(M)$ shows that $L$ acts on $Y \otimes \mathbb{Q}$ and that $L$ embeds into $\operatorname{End}^{0}(G)$. It follows (for example) from [Milne 1986, 3.9] that any morphism of the torus $T$ to an abelian variety is trivial. This implies that we have an embedding $L \subset \operatorname{End}^{0}(T)$ so $L$ also acts on $Y^{\star} \otimes \mathbb{Q}$ where $Y^{\star}=\operatorname{Hom}\left(T, \mathbb{G}_{m}\right)$. Finally, by passing to the quotient $A=G / T$, we obtain an embedding $L \subset \operatorname{End}^{0}(A)$.

By functoriality, $L$ acts on $V_{\ell}=V_{\ell}(M)$, making it into an $L \otimes_{\mathbb{Q}} \mathbb{Q}_{\ell}$-module. To ease notation, we will write $L_{\ell}=L \otimes_{\mathbb{Q}} \mathbb{Q}_{\ell}$ from now on. The weight filtration of $M$ (see [Raynaud 1994, 2.2]) induces an increasing $L_{\ell}$-linear filtration of $V_{\ell}$ such that the nonzero components of the associated graded are

$$
\begin{aligned}
\mathrm{Gr}_{-2}\left(V_{\ell}\right) & \cong\left(Y^{\star}\right)^{\vee} \otimes_{\mathbb{Z}} \mathbb{Q}_{\ell}(1), \\
\operatorname{Gr}_{-1}\left(V_{\ell}\right) & \cong V_{\ell}(A)=T_{\ell}(A) \otimes_{\mathbb{Z}_{\ell}} \mathbb{Q}_{\ell}, \\
\operatorname{Gr}_{0}\left(V_{\ell}\right) & \cong Y \otimes_{\mathbb{Z}} \mathbb{Q}_{\ell} .
\end{aligned}
$$

The action of $\Gamma_{F}$ respects the weight filtration and commutes with the $L_{\ell}$-action so $\Gamma_{F}$ acts $L_{\ell}$-linearly on $\mathrm{Gr}_{\bullet}\left(V_{\ell}\right)$. The isomorphisms above are $L_{\ell}$-linear and $\Gamma_{F}$-equivariant. One has $L_{\ell} \cong \bigoplus_{\lambda} L_{\lambda}$, where $\lambda$ runs through the primes of $L$ lying 
over $\ell$. This decomposition gives rise to corresponding decompositions of each $L_{\ell}$-module occurring above as a direct sum of $L_{\lambda}$-modules of the same rank.

The next aim is to establish a common $\mathbb{Q}$-structure $V$ on the modules $V_{\ell}$, endowed with $L$-action and weight filtration. We first describe the associated graded. For $V^{0}=Y \otimes_{\mathbb{Z}} \mathbb{Q}$, there is a system of canonical $L_{\ell}$-linear isomorphisms $\mathrm{Gr}_{0}\left(V_{\ell}\right) \cong V^{0} \otimes \mathbb{Q}_{\ell}$. Next, put $V^{-2}=\left(Y^{\star} \otimes_{\mathbb{Z}} \mathbb{Q}^{\vee}\right)^{\vee}$ and fix isomorphisms $\mathbb{Q}_{\ell}(1) \cong \mathbb{Q}_{\ell}$ for each $\ell$. This gives rise to a system of isomorphisms $\mathrm{Gr}_{-2}\left(V_{\ell}\right) \cong V^{-2} \otimes \mathbb{Q}_{\ell}$, depending only on the identifications $\mathbb{Q}_{\ell}(1) \cong \mathbb{Q}_{\ell}$.

We finally consider $\mathrm{Gr}_{-1}$. By the theorem of the primitive element, $L=\mathbb{Q}(\alpha)$, so it follows from [Mumford 1970, $\$ 19$, Theorem 4] that there exists an $L$-vector space $V^{-1}$ endowed with $L_{\ell}$-linear isomorphisms

$$
\mathrm{Gr}_{-1}\left(V_{\ell}\right) \cong V^{-1} \otimes_{\mathbb{Q}} \mathbb{Q}_{\ell}
$$

for every $\ell$; see [Noot 2006, proof of 6.13]. As a vector space is determined up to isomorphism by its dimension, $V^{-1}$ is unique up to $L$-linear isomorphisms and each isomorphism $\mathrm{Gr}_{-1}\left(V_{\ell}\right) \cong V^{-1} \otimes_{\mathbb{Q}} \mathbb{Q}_{\ell}$ is unique up to $L_{\ell}$-linear automorphisms of $\mathrm{Gr}_{-1}\left(V_{\ell}\right)$.

Define

$$
V=V^{-2} \oplus V^{-1} \oplus V^{0}
$$

endowed with the natural $L$-action and the increasing filtration defined by the grading. As $L_{\ell}$ is a semisimple algebra, $V_{\ell}$ is $L_{\ell}$-isomorphic to its associated graded, so the preceding discussion gives rise to a noncanonical $L_{\ell}$-linear isomorphism $V_{\ell} \cong V \otimes_{\mathbb{Q}} \mathbb{Q}_{\ell}$ for every $\ell$. We proved the following lemma.

Lemma 1.2. There exists an $L$-vector space $V$ endowed with an increasing filtration and, for each $\ell$, an $L_{\ell}$-linear isomorphism $V_{\ell} \cong V \otimes_{\mathbb{Q}} \mathbb{Q}_{\ell}$ compatible with the filtrations. In particular, each $V_{\ell}$ is a free $L_{\ell}$-module whose rank is independent of $\ell$ and the weight filtration is a filtration by free $L_{\ell}$-submodules of ranks independent of $\ell$.

1.3. The group $\boldsymbol{H}$. We fix an $L$-vector space $V$ together with a system of isomorphisms as in the lemma. Let $H=\operatorname{Res}_{L / \mathbb{Q}} \mathrm{GL}_{/ L}(V)$ be the linear algebraic group over $\mathbb{Q}$ of $L$-linear automorphisms of $V$ and let $\mathfrak{h}$ be its Lie algebra. This means that $\mathfrak{h}=\mathfrak{g l}_{L}(V)$ is the $\mathbb{Q}$-Lie algebra of $L$-linear endomorphisms of $V$. Obviously, $H$ acts on $\mathfrak{h}$ through the adjoint representation. By means of the identifications above, $H_{/ \mathbb{Q}_{\ell}}$ and $\mathfrak{h} \otimes_{\mathbb{Q}} \mathbb{Q}_{\ell}$ act on $V_{\ell}$. This identifies $H_{/ \mathbb{Q}_{\ell}}$ with the group of $L_{\ell}$-linear automorphisms of $V_{\ell}$ and $\mathfrak{h} \otimes \mathbb{Q}_{\ell}$ with its Lie algebra. These identifications are determined up to inner automorphisms for the groups and up to the adjoint action of $H_{/ \mathbb{Q}_{\ell}}$ where the Lie algebras are concerned. Finally note that $L=\operatorname{End}_{H}(V)$ and that $L_{\ell}=\operatorname{End}_{H_{/ \mathbb{Q}_{\ell}}}\left(V_{\ell}\right)$. 
1.4. The monodromy operator. From now on we assume that the 1-motive $M$ is strict in the sense of [Raynaud 1994, Définition 4.2.3], which means that the semiabelian variety $G$ has potentially good reduction. In this case, [ibid., 4.3] defines the geometric monodromy, a canonical additive map $\mu: Y \otimes Y^{\star} \rightarrow \mathbb{Q}$. Giving $\mu$ is equivalent to giving the induced map

$$
N: V^{0}=Y \otimes \mathbb{Q} \rightarrow V^{-2}=\left(Y^{\star} \otimes \mathbb{Q}\right)^{\vee} .
$$

By functoriality this map is $L$-linear, so we can interpret $N$ as an element of the Lie algebra $\mathfrak{h}^{\text {ss }} \subset \operatorname{End}(V)$. As an endomorphism of $V$, it is nilpotent of echelon 2 . In what follows, the notation $N$ will be reserved for this element of $\mathfrak{h}^{\text {ss }}$.

The map $N$ defines a morphism $\mathrm{Gr}_{0}\left(V_{\ell}\right) \rightarrow \mathrm{Gr}_{-2}\left(V_{\ell}\right)(-1)$ and thus a morphism $N_{\ell}: V_{\ell} \rightarrow V_{\ell}(-1)$. As $N_{\ell}$ is $L_{\ell}$-linear, $N_{\ell} \in \mathfrak{h}^{\mathrm{ss}} \otimes \mathbb{Q}_{\ell}(-1)$ for each $\ell$. Recall that the identification $V_{\ell} \cong V \otimes \mathbb{Q}_{\ell}$ depends on the identification $\mathbb{Q}_{\ell}(1) \cong \mathbb{Q}_{\ell}$ fixed in Section 1.1. Using the same identification, we identify $\mathfrak{h}^{\mathrm{ss}} \otimes \mathbb{Q}_{\ell}(-1)$ with $\mathfrak{h}^{\mathrm{ss}} \otimes \mathbb{Q}_{\ell}$ and under these isomorphisms the images of $N \otimes 1$ and $N_{\ell}$ in $\mathfrak{h}^{\mathrm{ss}} \otimes \mathbb{Q}_{\ell}$ coincide.

The discussion above only depends on the isomorphisms $\mathrm{Gr}_{i}\left(V_{\ell}\right) \cong V^{i} \otimes \mathbb{Q}_{\ell}$ for $i=-2,0$, which in turn depend only on the choice of an identification $\mathbb{Q}_{\ell}(1) \cong \mathbb{Q}_{\ell}$. In what follows we may thus change the splitting of the weight filtration and the identification $\mathrm{Gr}_{-1}\left(V_{\ell}\right) \cong V^{-1} \otimes \mathbb{Q}_{\ell}$ without affecting the properties above. We have established the following proposition.

Proposition 1.5. Let notation and assumptions be as above, in particular the motive $M$ is assumed to be strict and $N_{\ell}: V_{\ell} \rightarrow V_{\ell}(-1)$ is the $\ell$-adic monodromy operator. For each $\ell$, fix an identification of $H_{/ \mathbb{Q}_{\ell}}$ with the group of $L_{\ell}$-linear automorphisms of $V_{\ell}$ and an isomorphism $\mathbb{Q}_{\ell} \cong \mathbb{Q}_{\ell}(1)$. Using these identifications we consider $N_{\ell}$ as an element of $\mathfrak{h} \otimes \mathbb{Q}_{\ell}$.

- For every algebraically closed field $\Omega \supset \mathbb{Q}_{\ell}$ and every $\sigma \in \operatorname{Aut}(\Omega)$, the image of $N_{\ell}$ in $\left(\mathfrak{h} \otimes_{\mathbb{Q}} \mathbb{Q}_{\ell}\right) \otimes_{\mathbb{Q}_{\ell}} \Omega=\mathfrak{h} \otimes \Omega$ is conjugate to $\sigma\left(N_{\ell}\right)$ under the adjoint action of $H(\Omega)$.

- If $\Omega$ is an algebraically closed field containing both $\mathbb{Q}_{\ell}$ and $\mathbb{Q}_{\ell^{\prime}}$ then the images in $\mathfrak{h} \otimes \Omega$ of $N_{\ell}$ and $N_{\ell^{\prime}}$ are $H(\Omega)$-conjugate.

1.6. The action of inertia. The $\Gamma_{F}$-action on each $V_{\ell}$ is $L_{\ell}$-linear so the realisations of $M$ give rise to a system of representations

$$
\rho_{\ell}: \Gamma_{F} \rightarrow H\left(\mathbb{Q}_{\ell}\right),
$$

using the identifications from Section 1.1 and the group $H$ from Section 1.3.

Following [Deligne 1973, §2] for the basic notation, we discuss the action of the inertia group $I_{F} \subset \Gamma_{F}$. Let $v$ be the valuation of $F$ with value group $\mathbb{Z}$ and let $k$ be the (finite) residue field. We will write $\bar{v}$ for the valuation on $\bar{F}$ extending $v$ and write $\bar{k}$ for the residue field of $\bar{F}$. 
Let

$$
\widehat{\mathbb{Z}}_{\neq p}=\lim _{p \nmid n} \mathbb{Z} / n \mathbb{Z}=\prod_{\ell \neq p} \mathbb{Z}_{\ell}
$$

be the $p$-primary part of $\widehat{\mathbb{Z}}$ and let $\mathbb{A}_{\neq p}=\widehat{\mathbb{Z}}_{\neq p} \otimes_{\mathbb{Z}} \mathbb{Q}$. The prime-to- $p$ part of $\mathbb{Q} / \mathbb{Z}$ is $\mathbb{A}_{\neq p} / \widehat{\mathbb{Z}}_{\neq p}=(\mathbb{Q} / \mathbb{Z})_{\neq p}$. For every $n$ with $p \nmid n$, we identify $\left(\frac{1}{n} \mathbb{Z} / \mathbb{Z}\right)(1)$ with the group of $n$-th roots of unity in $\bar{k}$. This identifies $(\mathbb{Q} / \mathbb{Z})_{\neq p}(1)$ with the multiplicative group $\bar{k}^{\times}$. There is a natural morphism $t: I_{F} \rightarrow \widehat{\mathbb{Z}}_{\neq p}(1)$ such that $\sigma(x) x^{-1}=[t(\sigma) \bar{v}(x)]$ for all $\sigma \in I_{F}$ and $x \in \bar{F}^{\times}$. Here $[t(\sigma) \bar{v}(x)]$ is the image in $\bar{k}^{\times} \cong(\mathbb{Q} / \mathbb{Z})_{\neq p}(1)$ of $t(\sigma) \bar{v}(x) \in \mathbb{A}_{\neq p}(1)$. For $\ell \neq p$, we write $t_{\ell}: I_{F} \rightarrow \mathbb{Z}_{\ell}(1)$ for the composite of $t$ with the projection $\widehat{\mathbb{Z}}_{\neq p}(1) \rightarrow \mathbb{Z}_{\ell}(1)$.

In the case where $Y$ and $G$ have good reduction, it follows from [Raynaud 1994, Proposition 4.6.1] that if $\ell \neq p$ then for each $\sigma \in I_{F}$ one has

$$
\rho_{\ell}(\sigma)=\exp \left(N_{\ell} \otimes t_{\ell}(\sigma)\right)
$$

where $N_{\ell} \in \mathfrak{h}^{\text {ss }} \otimes \mathbb{Q}_{\ell}(-1)$ is the $\ell$-adic monodromy operator defined in Section 1.4. For an arbitrary strict 1-motive, the equality above holds for all $\sigma$ in a sufficiently small open subgroup of $I_{F}$. We finally note that $(1.6 *)$ characterises the operator $N_{\ell}$ as a map $N_{\ell}: V_{\ell} \rightarrow V_{\ell}(-1)$. This will play an important role in Section 2.2, in particular in the formula $(2.2 *)$.

1.7. Characteristic polynomials. Write $q=|k|$ and let $\varphi$ be the arithmetic Frobenius automorphism $\varphi: x \mapsto x^{q}$ of $\bar{k}$ over $k$. The Weil group of $F$ is the subgroup of $\Gamma_{F}$ consisting of the elements $\psi$ inducing an integral power of $\varphi^{\alpha(\psi)}$ of $\varphi$. The map $\alpha: W_{F} \rightarrow \mathbb{Z}$ thus defined is a group homomorphism and its kernel is the inertia group $I_{F} \subset \Gamma_{F}$. We endow the Weil group with the topology determined by the condition that $I_{F} \subset W_{F}$ is an open subgroup carrying the topology inherited from its topology as a Galois group.

For a 1-motive $M / F$ with $L$-action as before, $k=-2,-1$ or 0 and $\psi \in W_{F}$, let

$$
P_{L_{\ell}, \psi}^{(k)} \in L_{\ell}[T]
$$

be the characteristic polynomial of $\rho_{\ell}(\psi)$ acting as an $L_{\ell}$-linear endomorphism on the free $L_{\ell}$-module $\mathrm{Gr}_{k}\left(V_{\ell}\right)$. Let $P_{L_{\ell}, \psi}$ be the characteristic polynomial of $\rho_{\ell}(\psi)$ acting $L_{\ell}$-linearly on $V_{\ell}$. Obviously, one has

$$
P_{L_{\ell}, \psi}=\prod_{k=-2}^{0} P_{L_{\ell}, \psi}^{(k)} .
$$

Proposition 1.8. Let notation and hypotheses be as above; in particular $M$ is assumed to be strict. Let $\ell$ run though the primes different from $p$. Then for each $k=-2,-1,0$ and any $\psi \in W_{F}$, we have $P_{L_{\ell}, \psi}^{(k)} \in L[T]$. For fixed $k$ and $\psi$, the 
polynomial $P_{L_{\ell}, \psi}^{(k)}$ is independent of $\ell$ and all its complex roots have absolute value $q^{-\alpha(\psi) k / 2}$. The polynomial $P_{L_{\ell}, \psi}$ belongs to $L[T]$ and is independent of $\ell$.

Proof. It is sufficient to prove the statements concerning the $P_{L_{\ell}, \psi}^{(k)}$. For $k=-2,0$, these follow from the $\Gamma_{F}$-equivariant isomorphisms $\mathrm{Gr}_{-2}\left(V_{\ell}\right) \cong\left(Y^{\star}\right)^{\vee} \otimes \mathbb{Q}_{\ell}(1)$ and $\mathrm{Gr}_{0}\left(V_{\ell}\right) \cong Y \otimes \mathbb{Q}_{\ell}$ and the fact that $\Gamma_{F}$ acts on $Y$ and on $Y^{\star}$ through finite quotients.

For $k=-1$ we have $\mathrm{Gr}_{-1}\left(V_{\ell}\right) \cong V_{\ell} A$, where $A$ is an abelian variety with $L \subset \operatorname{End}^{0}(A)$. The statement about the absolute values of the roots of $P_{L_{\ell}, \psi}^{(-1)}$ therefore follows from the corollary to Theorem 3 in [Serre and Tate 1968]; see also [Raynaud 1994, 4.7.4]. Under the assumption that $A$ has good reduction and that $\psi$ is a Frobenius element, a proof of the claims that $P_{L_{\ell}, \psi}^{(-1)} \in L[T]$ and that this polynomial is independent of $\ell$ is sketched in [Noot 2009, 2.1]. Taking into account [Serre and Tate 1968, Theorem 2], the argument remains valid when $A$ only has potentially good reduction and $\psi \in I_{F}$. For the case where $\psi$ reduces to a nontrivial power of the Frobenius element, one replaces the use of [Serre and Tate 1968, Theorem 2] by the corollary to Theorem 3 in the same paper.

1.9. Remark. The action of any $\psi \in W_{F}$ on $\operatorname{Gr}_{k}\left(V_{\ell}\right)$ is semisimple for any $k$. For $k=-2,0$ this results from the fact that $\Gamma_{F}$ acts on $\mathrm{Gr}_{0}\left(V_{\ell}\right)$ and on $\mathrm{Gr}_{-2}\left(V_{\ell}\right)(-1)$ through a finite quotient. For $k=-1$ it follows from the fact that $\mathrm{Gr}_{-1}\left(V_{\ell}\right) \cong V_{\ell} A$, where $A$ is an abelian variety over $F$ with potentially good reduction. Combining this statement with the Proposition 1.8, it follows that each $\psi \in W_{F}$ with $\alpha(\psi) \neq 0$ acts semisimply on $V_{\ell}$.

1.10. Frobenius weights. As before, $M$ is a strict 1 -motive over $F$ with $L$-action. We fix an arithmetic Frobenius element $\Phi \in \Gamma_{F}$, that is, a lifting of the Frobenius automorphism $\varphi$ of $\bar{k}$; see Section 1.7. The operator $N_{\ell}: V_{\ell} \rightarrow V_{\ell}(-1)$ defined in Section 1.4 is $\Gamma_{F}$-equivariant, which implies that $\operatorname{Ad}\left(\rho_{\ell}(\Phi)\right)\left(N_{\ell}\right)=q N_{\ell}$.

As noted in Section 1.9, the image $\rho_{\ell}(\Phi)$ is semisimple and by Proposition 1.8 its eigenvalues are algebraic integers and, for any eigenvalue, all complex absolute values coincide and are equal to $q, q^{1 / 2}$ or 1 . For $k=-2,-1,0$, let $V_{\ell}^{k} \otimes \overline{\mathbb{Q}}_{\ell}$ be the sum of the eigenspaces associated to the eigenvalues with absolute value $q^{-k / 2}$. This defines a splitting $V_{\ell}=V_{\ell}^{-2} \oplus V_{\ell}^{-1} \oplus V_{\ell}^{0}$ of the weight filtration. The Frobenius weight cocharacter $w_{\ell}: \mathbb{G}_{m / \mathbb{Q}_{\ell}} \rightarrow H_{/ \mathbb{Q}_{\ell}}$ is the morphism making $\mathbb{G}_{m / \mathbb{Q}_{\ell}}$ act on $V_{\ell}^{k}$ through the $(k+1)$-st power map. The reader should take note of the shift in filtration, which is introduced to simplify matters later on. Through the adjoint representation, $w_{\ell}(t)$ acts on the line in $\mathfrak{h}^{\mathrm{ss}} \otimes \mathbb{Q}_{\ell}(-1)$ generated by $N_{\ell}$ as multiplication by $t^{-2}$.

If $M / F$ is any, not necessarily strict, 1 -motive with $L$-action, then the complex absolute values of any eigenvalue of $\rho_{\ell}(\Phi)$ are still equal to $q, q^{1 / 2}$ or 1 . This 
follows from the existence [Raynaud 1994, 4.2.2] of a strict 1-motive $M^{\prime}$ with $L$-action endowed with a system of canonical isomorphisms $V_{\ell}(M) \cong V_{\ell}\left(M^{\prime}\right)$. The Frobenius weight cocharacter $w_{\ell}$ of $M$ can therefore be defined exactly as before. It corresponds to the cocharacter associated to $M^{\prime}$ by transport via the isomorphism $V_{\ell}(M) \cong V_{\ell}\left(M^{\prime}\right)$ above. In general, $w_{\ell}$ does not split the weight filtration.

Finally note that, for $M$ strict, the identification $V_{\ell} \cong V \otimes \mathbb{Q}_{\ell}$ can be modified, without affecting its previously established properties, to ensure that the grading on $V_{\ell}$ defined by the Frobenius weights corresponds to the grading on $V$.

\section{The representations of the Weil-Deligne group associated to a 1-motive}

2.1. The Weil-Deligne group. In addition to the conventions in Section 1.1, we will from now on assume that $\ell, \ell^{\prime} \neq p$. As in Section 1.7, $W_{F}$ is the Weil group of $F$. We briefly summarise some of the notions introduced in [Deligne 1973, §8]; see also [Fontaine 1994].

Letting $\psi \in W_{F}$ operate on the additive group $\mathbb{G}_{a / \mathbb{Q}}$ as multiplication by $q^{\alpha(\psi)}$, one defines an action of the constant topological group scheme $W_{F}$ on $\mathbb{G}_{a / \mathbb{Q}}$. The Weil-Deligne group of $F$ is the semidirect product

$$
{ }^{\prime} W_{F}=W_{F} \ltimes \mathbb{G}_{a}
$$

defined by this action, viewed as a group scheme over $\mathbb{Q}$.

Fix an identification $\mathbb{Q}_{\ell} \cong \mathbb{Q}_{\ell}(1)$ as in Section 1.1, an arithmetic Frobenius element $\Phi \in W_{F}$ as in Section 1.10 and consider the map $t_{\ell}$ from Section 1.6 as a morphism

$$
I_{F} \rightarrow \mathbb{Q}_{\ell}(1) \cong \mathbb{Q}_{\ell}=\mathbb{G}_{a}\left(\mathbb{Q}_{\ell}\right) .
$$

We define a system of $\ell$-adic representations of $W_{F}$ with values in ${ }^{\prime} W_{F}\left(\mathbb{Q}_{\ell}\right)$ by

$$
\psi \mapsto\left(\psi, t_{\ell}\left(\Phi^{-\alpha(\psi)} \psi\right)\right) \in\left(W_{F} \ltimes \mathbb{G}_{a}\right)\left(\mathbb{Q}_{\ell}\right) .
$$

For a field $E$ of characteristic 0 and a linear algebraic group $G_{/ E}$ over $E$, giving an algebraic representation $\left({ }^{\prime} W_{F}\right)_{/ E} \rightarrow G_{/ E}$ is equivalent to giving a pair $\left(\rho^{\prime}, N\right)$ where $\rho^{\prime}: W_{F} \rightarrow G_{/ E}(E)$ is a linear representation that is trivial on some open subgroup of $I_{F}$ and $N \in \operatorname{Lie}\left(G_{/ E}\right)$ is a nilpotent element satisfying the condition that

$$
\operatorname{Ad}\left(\rho^{\prime}(\psi)\right) N=q^{\alpha(\psi)} N
$$

for all $\psi \in W_{F}$. The representation of $\left({ }^{\prime} W_{F}\right) / E$ corresponding to the pair $\left(\rho^{\prime}, N\right)$ is given by $(\psi, x) \mapsto \rho^{\prime}(\psi) \exp (N x)$.

2.2. ' $W_{F}$ and $\ell$-adic Galois representations. Let $H_{/ \mathbb{Q}_{\ell}}$ be a $\mathbb{Q}_{\ell}$-linear algebraic group and $\rho_{\ell}: W_{F} \rightarrow H_{/ \mathbb{Q}_{\ell}}\left(\mathbb{Q}_{\ell}\right)$ a continuous representation. By Grothendieck's 
$\ell$-adic monodromy theorem (see [Deligne 1973, 8.2]), there exists a nilpotent element $N_{\ell}^{\prime} \in \mathfrak{h}^{\mathrm{ss}}(-1)=\operatorname{Lie}\left(H_{/ \mathbb{Q}_{\ell}}\right)^{\mathrm{ss}}(-1)$ such that

$$
\rho_{\ell}(\psi)=\exp \left(N_{\ell}^{\prime} t_{\ell}(\psi)\right)
$$

for all $\psi$ in a sufficiently small open subgroup of $I_{F}$; see (1.6*). One can therefore associate to $\rho_{\ell}$ a representation $\left(\rho_{\ell}^{\prime}, N_{\ell}^{\prime}\right)$ of ${ }^{\prime} W_{F}$ with values in $H_{/ \mathbb{Q}_{\ell}}$ as follows.

Using the identification $\mathbb{Q}_{\ell} \cong \mathbb{Q}_{\ell}(1)$ to interpret $N_{\ell}^{\prime}$ as an element of $\mathfrak{h}^{\text {ss }}$, one defines

$$
\rho_{\ell}^{\prime}(\psi)=\rho_{\ell}(\psi) \exp \left(-N_{\ell}^{\prime} t_{\ell}\left(\Phi^{-\alpha(\psi)} \psi\right)\right) .
$$

Composing the corresponding algebraic representation of ${ }^{\prime} W_{F}$ with the natural representation $W_{F} \rightarrow{ }^{\prime} W_{F}\left(\mathbb{Q}_{\ell}\right)$ defined above, one recovers $\rho_{\ell}$.

According to [Deligne 1973, 8.11], the geometric conjugacy class of $\left(\rho_{\ell}^{\prime}, N_{\ell}^{\prime}\right)$ is independent of the choices of $\Phi$ and of the identification $\mathbb{Q}_{\ell} \cong \mathbb{Q}_{\ell}(1)$ made in this construction.

2.3. Compatible systems of representations of ${ }^{\prime} W_{F}$. Let $H$ be a reductive algebraic group over $\mathbb{Q}$. For a fixed $\ell$, we say that a representation ${ }^{\prime} W_{F / \mathbb{Q}_{\ell}} \rightarrow H_{\mathbb{Q}_{\ell}}$ is defined over $\mathbb{Q}$ (as a representation with values in $H$ ) if for every algebraically closed field $\Omega \supset \mathbb{Q}_{\ell}$, the base extension ${ }^{\prime} W_{F / \Omega} \rightarrow H_{/ \Omega}$ is conjugate under $H(\Omega)$ to all its images under $\operatorname{Aut}_{\mathbb{Q}}(\Omega)$. In terms of the pair $\left(\rho_{\ell}^{\prime}, N_{\ell}^{\prime}\right)$, let

$$
\rho_{\ell}^{\prime} \otimes_{\mathbb{Q}_{\ell}} \Omega: W_{F} \rightarrow H_{/ \Omega}(\Omega)
$$

be the extension of scalars and let $N_{\ell}^{\prime} \otimes_{\mathbb{Q}_{\ell}} 1 \in\left(\mathfrak{h} \otimes_{\mathbb{Q}_{\mathbb{Q}}} \mathbb{Q}_{\ell}\right) \otimes_{\mathbb{Q}_{\ell}} \Omega=\mathfrak{h} \otimes \Omega$ be the image of $N_{\ell}^{\prime}$. Then the condition above is equivalent to the condition that for every $\sigma \in \operatorname{Aut}_{\mathbb{Q}}(\Omega)$ there is an element $g \in H(\Omega)$ such that

$$
\sigma\left(\rho_{\ell}^{\prime} \otimes_{\mathbb{Q}_{\ell}} \Omega\right)=g\left(\rho_{\ell}^{\prime} \otimes_{\mathbb{Q}_{\ell}} \Omega\right) g^{-1} \quad \text { and } \quad \sigma\left(N_{\ell}^{\prime} \otimes_{\mathbb{Q}_{\ell}} 1\right)=\operatorname{Ad}(g)\left(N_{\ell}^{\prime} \otimes_{\mathbb{Q}_{\ell}} 1\right) \text {. }
$$

We say that a family of representations ${ }^{\prime} W_{F / \mathbb{Q}_{\ell}} \rightarrow H_{/ \mathbb{Q}_{\ell}}$ is a compatible system of representations of ${ }^{\prime} W_{F}$ (with values in $H$ ) if for every pair $\left(\ell, \ell^{\prime}\right.$ ) and every algebraically closed field $\Omega$ containing $\mathbb{Q}_{\ell}$ and $\mathbb{Q}_{\ell^{\prime}}$, the base extensions to $\Omega$ of the $\ell$-adic and $\ell^{\prime}$-adic representations of ${ }^{\prime} W_{F}$ are $H(\Omega)$-conjugate. In terms of the pairs $\left(\rho_{\ell}^{\prime}, N_{\ell}^{\prime}\right)$ and $\left(\rho_{\ell^{\prime}}^{\prime}, N_{\ell^{\prime}}^{\prime}\right)$, this means that there is a $g \in H(\Omega)$ such that

$$
\rho_{\ell}^{\prime} \otimes_{\mathbb{Q}_{\ell}} \Omega=g\left(\rho_{\ell^{\prime}}^{\prime} \otimes_{\mathbb{Q}_{\ell^{\prime}}} \Omega\right) g^{-1} \quad \text { and } \quad N_{\ell}^{\prime} \otimes_{\mathbb{Q}_{\ell}} 1=\operatorname{Ad}(g)\left(N_{\ell^{\prime}}^{\prime} \otimes_{\mathbb{Q}_{\ell^{\prime}}} 1\right) \in \mathfrak{h} \otimes \Omega .
$$

The action of $H(\Omega)$ by conjugation factors through $H(\Omega) \rightarrow H^{\text {ad }}(\Omega)$ so $H(\Omega)$ conjugacy may be replaced by $H^{\text {ad }}(\Omega)$-conjugacy everywhere.

2.4. Application to 1-motives. We apply the discussion above to the system of $\ell$-adic representations $V_{\ell}(M)$ associated to a 1-motive $M$ with $L$ action. Let $M$ be as in Section 1.1 and, as was the case from Section 1.4 onward, continue to 
assume $M$ to be strict. The numbers $r=\operatorname{rank}(Y), r^{\star}=\operatorname{dim}(T)$ and $g=\operatorname{dim}(A)$ are as in Section 1.1 and we fix an $L$-vector space $V$ and a system of identifications $V_{\ell} \cong V \otimes \mathbb{Q}_{\ell}$ as in Lemma 1.2. Let the algebraic group $H=\operatorname{Res}_{L / \mathbb{Q}} \mathrm{GL}_{/ L}(V)$ be as in Section 1.3. For every $\ell$, the group $H_{/ \mathbb{Q}_{\ell}}$ identifies with the group of $L_{\ell}=L \otimes \mathbb{Q}_{\ell}$-linear endomorphisms of $V_{\ell}$. The action of $\Gamma_{F}$ on $V_{\ell}(M)$ is $L_{\ell}$-linear, so it provides us with an $\ell$-adic representation of ${ }^{\prime} W_{F}$ with values in $H_{/ \mathbb{Q}_{\ell}}$, that is, a system of pairs $\left(\rho_{\ell}^{\prime}, N_{\ell}^{\prime}\right)$, where $N_{\ell}^{\prime} \in \mathfrak{h} \otimes \mathbb{Q}_{\ell}=\operatorname{Lie}(H) \otimes \mathbb{Q}_{\ell}$ and $\rho_{\ell}^{\prime}: W_{F} \rightarrow H_{/ \mathbb{Q}_{\ell}}$.

Lemma 2.5. Let $M / F$ be any 1-motive with $L$ action. The Frobenius weight cocharacter $w_{\ell}$ commutes with the representation $\rho_{\ell}^{\prime}$.

Proof. By construction, $\rho_{\ell}(\Phi)=\rho_{\ell}^{\prime}(\Phi)$ and the same equality holds for all powers of $\Phi$. In the construction of the Frobenius weight cocharacter, one may replace the Frobenius element $\Phi$, and hence $q$, by any strictly positive power without modifying $w_{\ell}$. This means that it is sufficient to prove that there is a strictly positive power $\Phi^{n}$ such that $\rho_{\ell}^{\prime}\left(\Phi^{n}\right)$ lies in the centre of the image of $\rho_{\ell}^{\prime}$. This is obvious since $\rho_{\ell}^{\prime}$ factors through an extension of the group generated by $\Phi$ by a finite quotient of $I_{F}$.

Proposition 2.6. Assume that we are in the situation of Section 2.4, so in particular $M$ is strict. Each $\ell$-adic representation

$$
{ }^{\prime} W_{F / \mathbb{Q}_{\ell}} \rightarrow H_{/ \mathbb{Q}_{\ell}}
$$

is defined over $\mathbb{Q}$ and these representations form a compatible system of representations of ${ }^{\prime} W_{F}$ with values in $H$.

Proof. We first show that each representation is defined over $\mathbb{Q}$.

The operator $N_{\ell}^{\prime}$ is determined by the fact that it satisfies $(2.2 *)$ for all $\psi$ in a sufficiently small open subgroup of the inertia group $I_{F}$. The equality $(1.6 *)$ implies that the monodromy operator $N_{\ell}$ has the same property so we conclude that $N_{\ell}^{\prime}=N_{\ell}$.

It follows from Proposition 1.5 that for every $\Omega \supset \mathbb{Q}_{\ell}$ and $\sigma \in \operatorname{Aut}(\Omega)$ as in Section 2.3, $N_{\ell} \in \mathfrak{h} \otimes \mathbb{Q}_{\ell}$ is $H(\Omega)$-conjugate to $\sigma\left(N_{\ell}\right)$. Let $g \in H(\Omega)$ be such that $\sigma\left(N_{\ell}\right)=\operatorname{Ad}(g)\left(N_{\ell}\right)$. It is sufficient to show that $\rho_{\ell}^{\prime} \otimes \Omega$ and $g^{-1} \sigma\left(\rho_{\ell}^{\prime} \otimes \Omega\right) g$ are conjugate under the stabiliser of $N_{\ell}$ in $H(\Omega)$. By elementary representation theory (see [Deligne 1973, Proposition 8.9]), it suffices to show that the representation $\rho_{\ell}^{\prime}$ is semisimple and that, for every $\psi \in W_{F}$, the $L_{\ell}$-linear characteristic polynomials of $\rho_{\ell}^{\prime}(\psi)$ and of $g^{-1} \sigma\left(\rho_{\ell}^{\prime} \otimes \Omega\right) g$ acting on each $\operatorname{Gr}_{i}^{\text {mon }}\left(V_{\ell}\right)$ coincide. In fact, it is sufficient to prove that the traces coincide. Here $\operatorname{Gr}_{i}^{\mathrm{mon}}\left(V_{\ell}\right)$ is the associated graded of $V_{\ell}$ for the monodromy filtration defined by $N_{\ell}$.

We first treat the semisimplicity. The restriction $\left.\rho_{\ell}^{\prime}\right|_{I_{F}}$ is semisimple because it factors through a finite quotient of $I_{F}$. As the action of $W_{F}$ on each $\mathrm{Gr}_{i}\left(V_{\ell}\right)$ is semisimple, the semisimplicity of $\rho_{\ell}^{\prime}$ results from Section 1.9 applied to any 
$\psi \in W_{F}$ with $\alpha(\psi) \neq 0$ and the fact that $W_{F}$ is an extension of the group generated by the Frobenius element to $\Phi$ by the inertia group $I_{F}$.

To establish the putative equality of the characteristic polynomials we will prove that the $L_{\ell}$-linear characteristic polynomials of the $\rho_{\ell}^{\prime}(\psi)$ acting on the $\operatorname{Gr}_{i}^{\text {mon }}\left(V_{\ell}\right)$ lie in $L[T]$. By Proposition 1.8 the corresponding statement is true for the action of $W_{F}$ on the $\mathrm{Gr}_{i}\left(V_{\ell}\right)$, the associated graded for the weight filtration. We finish the argument by passing to the graded for the monodromy filtration.

This is accomplished by considering the filtration

$$
W_{-2}^{\text {mon }} V_{\ell}=\operatorname{im}\left(N_{\ell}\right) \subset W_{-2} V_{\ell} \subset W_{-1} V_{\ell} \subset W_{-1}^{\text {mon }} V_{\ell}=\operatorname{ker}\left(N_{\ell}\right) \subset W_{0} V_{\ell}=V_{\ell} .
$$

The isomorphism $\mathrm{Gr}_{0}\left(V_{\ell}\right) \cong V^{0} \otimes \mathbb{Q}_{\ell}$ is $\Gamma_{F}$-equivariant and the action of $\Gamma_{F}$ on $V^{0} \otimes \mathbb{Q}_{\ell}$ comes from its $L$-linear action on $V^{0}$. Similarly, the action of $\Gamma_{F}$ on $\mathrm{Gr}_{-2}\left(V_{\ell}\right) \cong V^{-2} \otimes \mathbb{Q}_{\ell}(1)$ comes from its $L$-linear action on $V^{-2}$ and the cyclotomic action on $\mathbb{Q}_{\ell}(1)$. Finally, $N_{\ell}$ comes from the $L$-linear map $N: V^{0} \rightarrow V^{-2}$ so by (2.1*), the kernel of $N$ in $V^{0}$ and the image of $N$ in $V^{-2}$ are $W_{F}$-invariant $L$-linear subspaces. The representation induced by $\rho_{\ell}^{\prime}$ on each of the spaces

$$
\begin{array}{ll}
\mathrm{Gr}_{-2}^{\mathrm{mon}} V_{\ell}=\operatorname{im}\left(N_{\ell}\right) \subset \mathrm{Gr}_{-2} V_{\ell}, & W_{-2} V_{\ell} / \operatorname{im}\left(N_{\ell}\right) \cong \mathrm{Gr}_{-2} V_{\ell} / \mathrm{Gr}_{-2}^{\mathrm{mon}} V_{\ell}, \\
\operatorname{ker}\left(N_{\ell}\right) / W_{-1} V_{\ell} \subset \mathrm{Gr}_{0} V_{\ell}, \quad \text { and } \quad \operatorname{Gr}_{0}^{\mathrm{mon}} V_{\ell}=V_{\ell} / \operatorname{ker}\left(N_{\ell}\right),
\end{array}
$$

therefore, is a base extension of a representation of $W_{F}$ on an $L$-vector space. This proves the claim for $\mathrm{Gr}_{-2}^{\mathrm{mon}} V_{\ell}$ and $\mathrm{Gr}_{0}^{\mathrm{mon}} V_{\ell}$. For $\mathrm{Gr}_{-1}^{\mathrm{mon}} V_{\ell}$ the claim follows similarly by considering the graded for the filtration

$$
W_{-2} V_{\ell} / \operatorname{im}\left(N_{\ell}\right) \subset W_{-1} V_{\ell} / \operatorname{im}\left(N_{\ell}\right) \subset \operatorname{ker}\left(N_{\ell}\right) / \operatorname{im}\left(N_{\ell}\right)=\mathrm{Gr}_{-1}^{\mathrm{mon}} V_{\ell}
$$

The fact that the representations ' $W_{F / \mathbb{Q}_{\ell}} \rightarrow H_{/ \mathbb{Q}_{\ell}}$ form a compatible system is proved by an analogous argument. One now has to prove that, for $i=-2,-1,0$ and for each $\psi \in W_{F}$, the $L_{\ell}$-linear characteristic polynomials of $\rho_{\ell}^{\prime}(\psi)$ acting on $\mathrm{Gr}_{i}^{\mathrm{mon}}\left(V_{\ell}\right)$ are independent of $\ell$. Again by Proposition 1.8, this is true for the characteristic polynomials on the $\mathrm{Gr}_{i}\left(V_{\ell}\right)$. The $\ell$ independence of the characteristic polynomials on the $\mathrm{Gr}_{i}^{\mathrm{mon}}\left(V_{\ell}\right)$ follows from this by considering the combined filtration and adapting the argument above.

Corollary 2.7. Let $M$ be any 1-motive over $F$ with L-action. Then for each $\ell$, the $\ell$-adic realisation $V_{\ell}(M)$ is a free $L_{\ell}$-module. For $\ell \neq p$, the representations ${ }^{\prime} W_{F / \mathbb{Q}_{\ell}} \rightarrow H_{/ \mathbb{Q}_{\ell}}$ are defined over $\mathbb{Q}$ and form a compatible system of representations $o f^{\prime} W_{F}$ with values in $H$.

Proof. By Lemma 1.2, each $V_{\ell}(M)$ is a free $L_{\ell}$-module. This implies that $H_{/ \mathbb{Q}_{\ell}}$ identifies with the group of $L_{\ell}$-linear endomorphisms of $V_{\ell}(M)$ and that any two such identifications differ by an inner automorphism of $H_{/ \mathbb{Q}_{\ell}}$. It is therefore sufficient to prove the second statement for one system of such identifications. 
By [Raynaud 1994, 4.2.2] there are a strict 1-motive $M^{\prime}$ over $F$ and a system of canonical isomorphisms $V_{\ell}(M) \cong V_{\ell}\left(M^{\prime}\right)$ (for every $\ell$ ). Using these identifications and the remark above, the corollary follows from Proposition 2.6.

Corollary 2.8. With the notation and hypotheses of Corollary $2.7, \operatorname{ker}\left(\rho_{\ell}^{\prime}\right) \subset W_{F}$ is independent of $\ell$.

\section{Application to abelian varieties and statement of the main theorem}

We turn our attention to an abelian variety $A$ over a number field $F \subset \mathbb{C}$. If $F$ is sufficiently big, each $\ell$-adic representation associated to $A$ factors through $\rho_{\ell}: \Gamma_{F} \rightarrow G_{A}\left(\mathbb{Q}_{\ell}\right)$, where $G_{A}$ is the Mumford-Tate group of $A / \mathbb{C}$. For a fixed valuation $v$ of $F$, the construction sketched in Section 2.2 gives rise to a system of $\ell$-adic representations ' $W_{F_{v} / \mathbb{Q}_{\ell}} \rightarrow G_{A / \mathbb{Q}_{\ell}}$ of the Weil-Deligne group of $F_{v}$. It is hoped that these representations are defined over $\mathbb{Q}$ and that they form a compatible system of representations with values in $G_{A}$.

The statement of the main theorem is somewhat weaker; loosely speaking, it states that, after a finite extension of $F$, the representations of ${ }^{\prime} W_{F_{v}}$ form a compatible system when $G_{A}$ is replaced by a larger group of which $G_{A}$ is the identity component. As the construction will show, only certain factors of $G^{\mathrm{der}}$ of type $D$ are affected by this modification. In order to formulate the precise statement we need a number of constructions from the previous paper [Noot 2009].

3.1. Notation. From now on, $F \subset \mathbb{C}$ is a number field and $A / F$ an abelian variety. Let $\bar{F}$ be the algebraic closure of $F$ in $\mathbb{C}$ and $\Gamma_{F}=\operatorname{Gal}(\bar{F} / F)$ the absolute Galois group. We fix a valuation $\bar{v}$ of $\bar{F}$ and let $v$ be its restriction to $F$. Let $p$ be the residue characteristic of $v$ and $F_{v}$ the completion of $F$ at $v$. It is a finite extension of $\mathbb{Q}_{p}$. Let $\ell, \ell^{\prime} \neq p$ prime numbers.

3.2. Abelian varieties. Betti cohomology defines a fibre functor $H_{B}=H_{B}^{1}$ on the category of absolute Hodge motives generated by the motive of $A$ and the Tate motive $\mathbb{Q}(1)$. The Mumford-Tate $G_{A}$ of $A$ is the group of $\otimes$-automorphisms of this fibre functor; see [Noot 2009, 1.2] for a more detailed explanation. We will assume throughout that $G_{A}$ is connected, this condition holds after replacing $F$ by a finite extension and it implies that $G_{A}$ is the smallest linear algebraic $\mathbb{Q}$-group such that the Hodge structure on $\mathrm{H}_{\mathrm{B}}^{1}(A(\mathbb{C}), \mathbb{Q})$ is defined by a morphism $S=\operatorname{Res}_{\mathbb{C} / \mathbb{R}} \mathbb{G}_{m} \rightarrow G_{A / \mathbb{R}}$; see [ibid., 1.2]. Let $\mathfrak{g}_{A}$ be the Lie algebra of $G_{A}$.

For every $\ell$, the fibre functor $\mathrm{H}_{\text {ét, } \ell}$ defined by the $\ell$-adic étale cohomology is canonically isomorphic to $\mathrm{H}_{\mathrm{B}} \otimes \mathbb{Q}_{\ell}$. The representation of $\Gamma_{F}$ on the $\ell$-adic étale cohomology makes $\Gamma_{F}$ act on the functor $\mathrm{H}_{\text {ét, } \ell}$ and this gives rise to a morphism

$$
\rho_{\ell}: \Gamma_{F} \rightarrow G_{A}\left(\mathbb{Q}_{\ell}\right) .
$$


Let $\Phi_{v}$ an arithmetic Frobenius element, belonging to the decomposition group $\Gamma_{F_{v}} \cong D_{\bar{v}} \subset \Gamma_{F}$. This gives rise to local data as in Section 2.1. We consider the restriction of the $\rho_{\ell}$ to the Weil group $W_{v}=W_{F_{v}}$. As explained in Section 2.2, it defines a representation of the Weil-Deligne group ${ }^{\prime} W_{v}={ }^{\prime} W_{F_{v}}$, that is, a pair $\left(\rho_{\ell}^{\prime}, N_{\ell}^{\prime}\right)$ with $\rho_{\ell}^{\prime}: W_{v} \rightarrow G_{A}\left(\mathbb{Q}_{\ell}\right)$ and $N_{\ell}^{\prime} \in \mathfrak{g}_{A}^{\text {ss }} \otimes \mathbb{Q}_{\ell}$. By Corollary 2.8, there is an open subgroup $J$ of the inertia group $I_{\bar{v}}$ such that each $\rho_{\ell}^{\prime}$ is trivial on $J$.

To apply the results above on 1-motives, it is convenient to work with Tate modules instead of étale cohomology groups. The $\ell$-adic Galois representation $V_{\ell} A=T_{\ell} A \otimes_{\mathbb{Z}_{\ell}} \mathbb{Q}_{\ell}$ is dual to $\mathrm{H}_{\text {ét }}^{1}\left(A_{/ \bar{F}}, \mathbb{Q}_{\ell}\right)$. Identification of the fibre functor defined by $V_{\ell}$ to the dual of the one defined by $\mathrm{H}_{\text {ét, } \ell}$ endows $V_{\ell}$ with the structure of a representation of $G_{A / \mathbb{Q}_{\ell}}$. The action of $\Gamma_{F}$ on $V_{\ell} A$ is given by the same morphism $\rho_{\ell}: \Gamma_{F} \rightarrow G_{A}\left(\mathbb{Q}_{\ell}\right)$ as before. The corresponding representation $\left(\rho_{\ell}^{\prime}, N_{\ell}^{\prime}\right)$ of the Weil-Deligne group ' $W_{F_{v}}$ is also unchanged.

3.3. The group $\boldsymbol{G}^{\natural \text { ad }}$. In [Noot $\left.2009,1.5\right]$ one finds the construction of a group

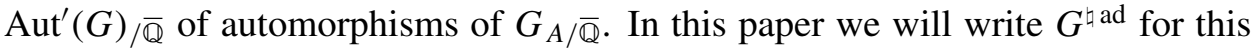
"natural extension" of $G^{\text {ad }}$. We briefly sketch its construction.

The derived group $G_{A / \bar{Q}}^{\mathrm{der}}$ is the almost direct product of almost simple subgroups $G_{i} \subset G_{A / \overline{\mathbb{Q}}}$, for $i$ in some index set $I$. Let $J \subset I$ be the set of indices $i$ such that $G_{i} \cong \operatorname{SO}\left(2 k_{i}\right) / \overline{\mathbb{Q}}$ for some $k_{i} \geq 4$ and for each $i \in J$ put $G_{i}^{\prime}=\mathrm{O}\left(2 k_{i}\right) \supset G_{i}$. We define

$$
G^{\natural \mathrm{ad}}=\prod_{i \in J} G_{i}^{\mathrm{ad}} \times \prod_{i \in I \backslash J} G_{i}^{\mathrm{ad}} \supset G_{A / \overline{\mathbb{Q}}}^{\mathrm{ad}} .
$$

As this group operates trivially on the centre of $G_{A / \bar{Q}}^{\text {der }}$, we can define an action of $G^{\natural \text { ad }}$ on $G_{A / \overline{\mathbb{Q}}}$ extending the adjoint action on $G_{A / \overline{\mathbb{Q}}}^{\text {der }}$ and with $G^{\natural \text { ad }}$ acting trivially on the centre of $G_{A / \overline{\mathbb{Q}}}$. Through the adjoint representation, the group $G^{\natural \text { ad }}$ also acts on the Lie algebra $\mathfrak{g} \otimes \overline{\mathbb{Q}}$.

3.4. Compatible systems revisited. We introduce a variant of the notion, introduced in Section 2.3, of a compatible system defined over $\mathbb{Q}$ of representations of ' $W_{F_{v}}$ with values in $G_{A}$. This time we allow conjugation by the group $G^{\natural \text { ad }}$ so the condition is weaker than $G$-conjugacy if $G_{A / \bar{Q}}^{\mathrm{der}}$ has factors of the form $\operatorname{SO}(2 k)$.

Let $v,{ }^{\prime} W_{v}$ and $\Phi_{v}$ be as in Sections 3.1 and 3.2. For a fixed $\ell$, let $\left(\rho_{\ell}^{\prime}, N_{\ell}^{\prime}\right)$ define a representation of ${ }^{\prime} W_{v / \mathbb{Q}_{\ell}}$ with values in $G_{A / \mathbb{Q}_{\ell}}$. We say that this representation is defined over $\mathbb{Q}$ modulo $G_{A}^{\natural \text { ad }}$ if, for every algebraically closed field $\Omega \supset \mathbb{Q}_{\ell}$ and every $\sigma \in \operatorname{Aut}_{\mathbb{Q}}(\Omega)$, there is a $g \in G_{A}^{\natural \text { ad }}(\Omega)$ such that

$$
\sigma\left(\rho_{\ell}^{\prime} \otimes_{\mathbb{Q}_{\ell}} \Omega\right)=g\left(\rho_{\ell}^{\prime} \otimes_{\mathbb{Q}_{\ell}} \Omega\right) g^{-1} \quad \text { and } \quad \sigma\left(N_{\ell}^{\prime} \otimes_{\mathbb{Q}_{\ell}} 1\right)=\operatorname{Ad}(g)\left(N_{\ell}^{\prime} \otimes_{\mathbb{Q}_{\ell}} 1\right) .
$$

We say that a system of representations $\left(\rho_{\ell}^{\prime}, N_{\ell}^{\prime}\right)$ of ${ }^{\prime} W_{v / \mathbb{Q}_{\ell}}$ is a compatible system of representations of ${ }^{\prime} W_{v}$ modulo $G_{A}^{\natural \text { ad }}$ if for every pair $\left(\ell, \ell^{\prime}\right)$ and every 
algebraically closed field $\Omega \supset \mathbb{Q}_{\ell}, \mathbb{Q}_{\ell^{\prime}}$, there is a $g \in G_{A}^{\natural \text { ad }}(\Omega)$ such that

$$
\rho_{\ell}^{\prime} \otimes_{\mathbb{Q}_{\ell}} \Omega=g\left(\rho_{\ell^{\prime}}^{\prime} \otimes_{\mathbb{Q}_{\ell^{\prime}}} \Omega\right) g^{-1} \quad \text { and } \quad N_{\ell}^{\prime} \otimes_{\mathbb{Q}_{\ell}} 1=\operatorname{Ad}(g)\left(N_{\ell^{\prime}}^{\prime} \otimes_{\mathbb{Q}_{\ell^{\prime}}} 1\right) \in \mathfrak{g} \otimes \Omega .
$$

Definition 3.5. Let $\Omega$ be an algebraically closed field, $G$ a linear algebraic group over a subfield of $\Omega$ and $V$ a representation of $G$. A semisimple $g \in G(\Omega)$ is neat if the Zariski closure of the subgroup of $G(\Omega)$ generated by $g$ is connected. A semisimple element $g \in G(\Omega)$ is weakly neat (with respect to $V$ ) if 1 is the only root of unity among the quotients $\lambda \mu^{-1}$ of eigenvalues $\lambda$ and $\mu$ of $g$.

For weak neatness, we will suppress the reference to $V$ if it is clear which representation is being considered. For elements of the Mumford-Tate group of an abelian variety, we always consider the representation defined by the Tate module. A neat element is weakly neat with respect to any representation.

Theorem 3.6. Assume that $A$ has semistable reduction at $v$ and that, for some $\ell$, the image $\rho_{\ell}^{\prime}\left(\Phi_{v}\right)$ is weakly neat. Then the representations $\left(\rho_{\ell}^{\prime}, N_{\ell}^{\prime}\right)$ of ${ }^{\prime} W_{v}$ corresponding to $A$ are defined over $\mathbb{Q}$ modulo the action $G_{A}^{\natural \text { ad }}$. For $\ell \neq p$, these representations form a compatible system of representations of ${ }^{\prime} W_{v}$ modulo the action of $G_{A}^{\natural \text { ad }}$.

\subsection{Remarks.}

3.7.1. The condition that $A$ has semistable reduction at $v$ implies that $\rho_{\ell}^{\prime}$ is trivial on $I_{\bar{v}}$. In particular, the condition that $\rho_{\ell}^{\prime}\left(\Phi_{v}\right)$ is weakly neat does not depend on the choice of the Frobenius element $\Phi_{v}$. Also note that $\rho_{\ell}^{\prime}\left(\Phi_{v}\right)=\rho_{\ell}\left(\Phi_{v}\right)$ so that the condition can also be checked on $\rho_{\ell}\left(\Phi_{v}\right)$. By the main theorem, or in a more elementary fashion by Proposition 1.8, the condition that $\rho_{\ell}^{\prime}\left(\Phi_{v}\right)$ is weakly neat is independent of $\ell$.

3.7.2. In general, $A$ only has potentially semistable reduction at $v$. In this case, $\left.\rho_{\ell}^{\prime}\right|_{I_{\bar{v}}}$ has finite image so, for $\sigma \in I_{\bar{v}}$, all eigenvalues of $\rho_{\ell}^{\prime}(\sigma)$ are roots of unity. The elements of $\rho_{\ell}^{\prime}\left(I_{\bar{v}}\right)$ are not neat and the methods of this paper do not seem to permit one to prove that the $\rho_{\ell}^{\prime}$ form a compatible system in this case. As the monodromy operators are unchanged by a finite base extension, one may reduce to the case of stable reduction to prove that the $N_{\ell}^{\prime}$ do form a compatible system defined over $\mathbb{Q}$.

3.7.3. To check the condition of weak neatness, one has to determine the characteristic polynomial of $\rho_{\ell}\left(\Phi_{v}\right)$ for at least one $\ell$, which is not always feasible in practice. However, the condition always holds for a power of $\Phi_{v}$, that is, after replacing $F$ by a finite extension $F^{\prime}$ and $v$ by a valuation of $F^{\prime}$ lying over $v$. Also note that if, for some $\ell \neq p$, the elements of $\rho_{\ell}\left(\Gamma_{F}\right)$ are congruent to 1 modulo $\ell$ (or congruent to 1 modulo 4 if $\ell=2$ ), then $\rho_{\ell}\left(\Phi_{v}\right)$ is necessarily weakly neat.

On the other hand, it is easy to construct abelian varieties, even with good reduction, that do not satisfy the condition of weak neatness. For this, one may 
choose a $p^{a}$-Weil number $\alpha$ having two conjugates differing by a nontrivial root of unity. There exists an abelian variety $A_{0}$ over a finite field of characteristic $p$ such that the characteristic polynomial of Frobenius is a power of the minimum polynomial of $\alpha$. Any lifting of $A_{0}$ over a number field provides a counterexample to the neatness condition of the theorem.

3.7.4. If the abelian variety $A$ has good reduction at $v$ then the monodromy $N_{\ell}^{\prime}$ is trivial and the theorem reduces to the main result, [Noot 2009, Théorème 1.8].

3.7.5. We finally refer to [ibid., Remark 1.9(4)] for a note on the density of the set of places $v$ of good reduction where $\rho_{\ell}\left(\Phi_{v}\right)$ is weakly neat. Density statements of this type are not useful in the present context as the number of places where $A$ does not have good reduction is finite.

3.8. $G_{A}$, monodromy and Frobenius weights. As pointed out in Section 3.2, the system $\left(\rho_{\ell}\right)$ is determined by the Galois representations on the Tate modules of $A$. From now on, we systematically adopt this point of view.

In the proof of Corollary 2.7, we applied [Raynaud 1994, 4.2] to the motive $M$ in order to reduce to a strict motive $M^{\prime}$. Applying the same argument to the abelian variety $A_{v}=A_{/ F_{v}}$, one again obtains a strict 1-motive $M^{\prime} / F_{v}$ endowed with a system of canonical $\Gamma_{F_{v}}$-equivariant isomorphisms

$$
V_{\ell}\left(A_{v}\right)=T_{\ell}\left(A_{v}\right) \otimes_{\mathbb{Z}_{\ell}} \mathbb{Q}_{\ell} \cong V_{\ell}\left(M^{\prime}\right) .
$$

Let $M^{\prime}=[Y \rightarrow G]$, let $Y^{\star}$ be the character group of the toric part of $G$ and write $r$ and $r^{\star}$ for the ranks of $Y$ and $Y^{\star}$. Let $g$ be the dimension of the quotient of $G$ by its maximal torus.

In Section 1.10 we defined the Frobenius weight cocharacter of a local Galois representation associated to a 1-motive. Applying this construction to the restrictions $\left.\rho_{\ell}\right|_{D_{v}}$ we obtain the Frobenius weight cocharacter

$$
w_{\ell}: \mathbb{G}_{m} \rightarrow \mathrm{GL}\left(V_{\ell}(A)\right) \text {. }
$$

Lemma 3.9. Under the conditions above we have $r=r^{\star}$ and the monodromy operator $N: Y \otimes \mathbb{Q} \rightarrow Y^{\star} \otimes \mathbb{Q}$ associated to $M^{\prime}$ is an isomorphism. For each $\ell$, the map $N_{\ell}^{\prime}$ defines an isomorphism from the $t$-eigenspace of $w_{\ell}$ acting on $V_{\ell}(A)$ onto the $t^{-1}$-eigenspace.

Proof. The arguments used in [Raynaud 1994, 4.2] show that $Y=\Lambda$, where $\Lambda$ is the $\mathbb{Z}$-module in the diagram (**) of [loc. cit.], so $r$ is equal to the rank of $\Lambda$. This reference also implies that $r^{\star}$ is equal to the rank of $\Lambda$, so $r=r^{\star}$. Still by [ibid., 4.2], the intersection of $\Lambda$ with the rigid analytic generic fibre of $G$ is trivial. With the notation of [ibid., 4.3], this means that for any $y \in Y$, there exists $y^{\star} \in Y^{\star}$ with 
$\mu_{o}\left(y \otimes y^{\star}\right)>0$. It follows that $N$ induces an isomorphism $Y \otimes \mathbb{Q} \cong Y^{\star} \otimes \mathbb{Q}$. All this is classical; see for example [Grothendieck et al. 1972, Exposé IX, Théorème 10.4].

The monodromy filtration on $V_{\ell}\left(M^{\prime}\right)$ coincides with the weight filtration so the last statement immediately follows from the previous ones.

Lemma 3.10. The Frobenius weight cocharacter $w_{\ell}$ factors through $G_{A / \mathbb{Q}_{\ell}}$. In fact, this cocharacter factors through a torus $T_{v} \subset G_{A / \mathbb{Q}_{\ell}}$ containing $\rho_{\ell}\left(\Phi_{v}\right)$.

Proof. The Mumford-Tate group $G_{A}$ contains the group $\mathbb{G}_{m / \mathbb{Q}}$ of scalar multiplications of $\mathrm{H}_{\mathrm{B}}^{1}(A(\mathbb{C}), \mathbb{Q})$. Let $w_{\ell}^{\prime}=t \cdot w_{\ell}$, let $T_{v}^{\prime} \subset G_{A / \mathbb{Q}_{\ell}}$ be the identity component of the Zariski closure of the subgroup of $G_{A / \mathbb{Q}_{\ell}}$ generated by $\rho_{\ell}\left(\Phi_{v}\right)$ and put $T_{v}=\mathbb{G}_{m} T_{v}^{\prime}$. We will prove that $w_{\ell}$ factors through $T_{v}$ by showing that $w_{\ell}^{\prime}$ factors through $T_{v}^{\prime}$.

The last statement follows from the argument used in [Serre 2000], \$4 of the first letter. The proof comes down to the fact that the eigenvalues of $w_{\ell}^{\prime}(t)$ satisfy all the multiplicative relations satisfied by the archimedean absolute values of the eigenvalues of $\rho_{\ell}\left(\Phi_{v}\right)$.

\section{Generalities on tractable abelian varieties}

4.1. Tractable abelian varieties. The notion of "variété abélienne accommodante" was introduced in [Noot 2009, 2.3]; in this paper we call such a variety a tractable abelian variety. Let us recall the relevant ideas.

First of all, we define the notion of an admissible representation of a reductive group. Heuristically, the admissible representations are the representations encountered when studying Shimura data of abelian type that admit an embedding into the Siegel Shimura datum. We refer to [Deligne 1979, 1.3] for this classification. To be precise, let $K$ be a field of characteristic 0 and let $\Omega \supset K$ be an algebraically closed extension. Assume that $G^{s}$ is a linear algebraic group over $K$ such that $G_{/ \Omega}^{s}$ is almost simple of type $A, B, C$ or $D$. Let $V^{s}$ be a faithful $K$-linear representation of $G^{s}$. We say that $V^{s}$ is an admissible representation of $G^{s}$ in the following cases:

- $G_{/ \Omega}^{s}$ is of type $A_{n}$ and $V^{s} \otimes_{K} \Omega$ is a multiple of the direct sum of the representations of highest weights $\varpi_{1}$ and $\varpi_{n}$ if $n \geq 2$ and a multiple of the representation of highest weight $\varpi_{1}$ if $n=1$.

- $G_{/ \Omega}^{s}$ is of type $B_{n}$ and $V^{s} \otimes_{K} \Omega$ is a multiple of the representation of highest weight $\varpi_{n}$.

- $G_{/ \Omega}^{s}$ is of type $C_{n}$ and $V^{s} \otimes_{K} \Omega$ is a multiple of the representation of highest weight $\varpi_{1}$.

- $G_{/ \Omega}^{s}$ is of type $D_{n}$ and $V^{s} \otimes_{K} \Omega$ is a multiple of the representation of highest weight $\varpi_{1}$. 
- $G_{/ \Omega}^{s}$ is of type $D_{n}$ and $V^{s} \otimes_{K} \Omega$ is a multiple of the direct sum of the representations of highest weights $\varpi_{n-1}$ and $\varpi_{n}$.

In the first three cases, we will say that the pair $\left(G^{s}, V^{s}\right)$ is of type $A_{n}, B_{n}$ or $C_{n}$, in the last two cases we say that $\left(G^{s}, V^{s}\right)$ is of type $D_{n}^{\sharp \mathbb{t}}$ or of type $D_{n}^{\mathbb{R}}$, respectively.

Returning to abelian varieties, we let $A$ be an abelian variety over $\mathbb{C}$ and write $V=\mathrm{H}_{\mathrm{B}}^{1}(A(\mathbb{C}), \mathbb{Q})$. We say that $A$ is strictly tractable if

- there exists a totally real number field $K$ and an almost simple linear algebraic group $G^{s}$ over $K$ such that $G_{A}^{\text {der }}=\operatorname{Res}_{K / \mathbb{Q}} G^{s}$;

- as a representation of $G_{A}^{\mathrm{der}}$, the cohomology group $V$ is the restriction of scalars of an admissible representation $V^{s}$ of $G^{s}$;

- if $\left(G^{s}, V^{s}\right)$ is of type $D_{n}^{\mathbb{R}}$ then every character space in $V \otimes \overline{\mathbb{Q}}$ for the action of the centre of $G_{A / \overline{\mathbb{Q}}}$ is an admissible representation of a factor of $G_{A / \overline{\mathbb{Q}}}^{\mathrm{der}}$; and

- the conditions above do not hold for any proper abelian subvariety of $A$.

The type of a strictly tractable abelian variety is the type of the pair $\left(G^{s}, V^{s}\right)$.

We will say that $A$ is tractable if $A$ is isogenous to a product $\prod_{i=1}^{m} A_{i}$ of strictly tractable abelian varieties $A_{i}$ and $G_{A}^{\mathrm{der}} \cong \prod_{i=1}^{m} G_{A_{i}}^{\mathrm{der}}$. If $F \subset \mathbb{C}$ is a subfield, an abelian variety $A / F$ is (strictly) tractable if $A_{/ \mathbb{C}}$ is and if $G_{A}$ is connected.

4.2. Remark. The concept of tractability is an auxiliary notion used in the proof of the main theorem. It does not seem to be of independent interest, though it is conceivable that the method of the present paper can be used in other contexts.

Heuristically, the fact that an abelian variety $A$ is strictly tractable means that the representation of $G_{A}^{\mathrm{der}}$ on $V=\mathrm{H}_{\mathrm{B}}^{1}(A(\mathbb{C}), \mathbb{Q})$ decomposes over $\overline{\mathbb{Q}}$ as a direct sum of irreducible representations of the almost simple factors of $G_{A / \overline{\mathbb{Q}}}^{\mathrm{der}}$. In particular, any abelian variety $A / \mathbb{C}$ of dimension $g$ with $G_{A}^{\mathrm{der}}=\mathrm{Sp}_{2 g}$ is tractable. This means that, in the moduli space of $g$-dimensional abelian varieties, the points corresponding to nontractable varieties belong to a countable union of closed subvarieties and thus the general abelian variety is tractable.

If the simple factors of $V \otimes \overline{\mathbb{Q}}$, as a representation of $G_{A / \overline{\mathbb{Q}}}^{\mathrm{der}}$, are tensor products of irreducible representations of the almost simple factors $G_{A / \overline{\mathbb{Q}}}^{\mathrm{der}}$, then $A$ is not tractable. The simplest such example was given by Mumford [1969]. The generic members of the families constructed there are not tractable.

More generally, any simple abelian variety $A / \mathbb{C}$, with $L=\operatorname{End}^{0}(A)$, whose Mumford-Tate group coincides with the group of $L$-linear symplectic similitudes of $\mathrm{H}_{\mathrm{B}}^{1}(A(\mathbb{C}), \mathbb{Q})$ is tractable. The converse is not true, as can be seen for example in the case where the Mumford-Tate group is of type $D_{n}$. Nevertheless, being tractable still means that the endomorphism algebra is big compared to the Mumford-Tate group. This is the key to the proof of the main theorem for tractable abelian varieties. 
By definition, abelian varieties of CM type are not tractable. As pointed out in the introduction, the system of $\ell$-adic representations associated to an abelian variety of CM type is described by the theory of complex multiplication and the main theorem is true in the CM case.

4.3. The algebra $L \subset \operatorname{End}^{0}(A)$. In the proof of the main Theorem 3.6, we will adapt the ideas used in [Noot 2009]. We will in particular make use of the algebra $L \subset \operatorname{End}(A) \otimes \mathbb{Q}$ constructed in the beginning of the proof of [ibid., Théorème 2.4]. For this construction, first decompose $V \otimes \overline{\mathbb{Q}}=\bigoplus_{i=1}^{n} V_{i}$, where the $V_{i}$ are the isotypic components of the representation of $G_{A / \overline{\mathbb{Q}}}$ on $V \otimes \overline{\mathbb{Q}}$. In other words, each $V_{i}$ is a multiple of an irreducible representation of $G_{A / \overline{\mathbb{Q}}}$ and $\operatorname{Hom}_{G_{A / \overline{\mathbb{Q}}}}\left(V_{i}, V_{j}\right)=0$ for $i \neq j$. This decomposition defines a subalgebra $\overline{\mathbb{Q}}^{n} \subset \operatorname{End}_{G_{A} / \overline{\mathbb{Q}}}(V \otimes \overline{\mathbb{Q}})$, with the $i$-th factor $\overline{\mathbb{Q}}$ acting on the factor $V_{i}$ by scalar multiplication. Taking $\Gamma_{\mathbb{Q}}$-invariants, this inclusion descends to

$$
L \subset \operatorname{End}_{G_{A / \overline{\mathbb{Q}}}}(V \otimes \overline{\mathbb{Q}})=\operatorname{End}^{0}(A / \mathbb{C})=\operatorname{End}^{0}(A),
$$

where $L$ is a finite, semisimple, commutative $\mathbb{Q}$-algebra. The last equality follows from the fact that $G_{A}$ is connected and is justified in [Noot 2009, proof of 2.4].

There is a canonical isomorphism $L \otimes \overline{\mathbb{Q}} \cong \prod_{l: L \rightarrow \overline{\mathbb{Q}}} \overline{\mathbb{Q}}$; in fact $L$ is defined as the algebra of $\Gamma_{\mathbb{Q}}$-invariants in the product on the right hand side. The direct factors of $V \otimes \overline{\mathbb{Q}}$ are indexed by the morphisms $\iota: L \rightarrow \overline{\mathbb{Q}}$, with the $\iota$-factor of $\prod_{\iota} \overline{\mathbb{Q}}$ acting on $V_{\iota}$ by scalar multiplications and acting trivially on the other $V_{\kappa}$. The decomposition of $L \otimes \overline{\mathbb{Q}}$ thus gives rise to a decomposition $V \otimes \overline{\mathbb{Q}}=\prod_{\iota} V_{\iota}$. There is a similar decomposition $V \otimes \Omega=\prod_{\iota} V_{\iota}$ for any algebraically closed field $\Omega$ of characteristic 0 , with the product taken over all morphisms $\iota: L \rightarrow \Omega$.

Lemma 4.4. Assume that $A / \mathbb{C}$ is a strictly tractable abelian variety. Unless $A$ is of type $D_{n}^{\mathbb{R}}$, the algebra above $L \subset \operatorname{End}(A) \otimes \mathbb{Q}$ is a field. If $A / \mathbb{C}$ of type $D_{n}^{\mathbb{R}}$ then the algebra $L$ is either a field or it is isomorphic to $L^{\prime} \times L^{\prime}$ for a field $L^{\prime}$.

Proof. As $L$ is a semisimple algebra, it decomposes as a product of fields. This decomposition gives rise to a corresponding decomposition of $A$ and unless the pair $\left(G^{s}, V^{s}\right)$ associated to $A$ is of type $A_{n}$ or $D_{n}^{\mathbb{R}}$, each factor still satisfies the first three conditions of the definition of a strictly tractable abelian variety. If there is more than one factor, this violates the minimality condition.

If the pair $\left(G^{s}, V^{s}\right)$ associated to $A$ is of type $A_{n}$, then the argument used in [Noot 2006, 5.1] shows that the complex conjugation acts on the Dynkin diagram of $G^{s}$ by the main involution. This implies that a direct factor of $V \otimes \overline{\mathbb{Q}}$, which is a representation of highest weight $\varpi_{1}$ of some factor of $G_{A / \overline{\mathbb{Q}}}^{\mathrm{der}}$ belongs to the same $\Gamma_{\overline{\mathbb{Q}}}$-orbit as the representation of highest weight $\varpi_{n}$ of the same factor. It follows that for any decomposition of $A$ as above, each factor still satisfies the first three 
conditions of the definition. The minimality condition again implies that there is only one factor.

If $\left(G^{s}, V^{s}\right)$ is of type $D_{n}^{\mathbb{R}}$, then a direct factor $L^{\prime}$ of $L$ may define a factor $A^{\prime}$ of $A$ such that the associated pair $\left(G^{s}, V^{s}\right)$ is a half spin representation. In that case, $A$ has a factor $A^{\prime \prime}$ for which $\left(G^{s}, V^{\prime s}\right)$ is the other half spin representation. By minimality, one must have $A \sim A^{\prime} \oplus A^{\prime \prime}$. In this case, the set of vertices $\varpi_{n-1}$ and the set of vertices $\varpi_{n}$ of the Dynkin diagram of $G_{A}^{\text {der }}$ form two separate orbits for the $\Gamma_{\mathbb{Q}}$ action. Since these orbits are isomorphic as $\Gamma_{\mathbb{Q}}$-sets, it follows that $L=L^{\prime} \oplus L^{\prime}$.

4.5. The group $\boldsymbol{H}$. For the rest of this section we will assume, in addition to the hypotheses of Section 3.1, that $A / F$ is tractable. Let $G_{A}^{\natural \text { ad }}$ be the linear algebraic group over $\overline{\mathbb{Q}}$ introduced in Section 3.3.

To prove the theorem for $A$, we will use the results on 1-motives obtained in Section 2 so it is convenient to study the Galois representations defined by the Tate-modules; see Section 3.2. Let $V=\mathrm{H}_{1}(A(\mathbb{C}), \mathbb{Q})$ and for each prime number $\ell$ put $V_{\ell}=T_{\ell}(A) \otimes_{\mathbb{Z}_{\ell}} \mathbb{Q}_{\ell}$, where $T_{\ell}(A)$ is the $\ell$-adic Tate module of $A$. As in Section 3.2, there is a natural representation of $G_{A}$ on $V$ and there are canonical isomorphisms $V_{\ell} \cong V \otimes \mathbb{Q}_{\ell}$. The action of $\Gamma_{F}$ on the $V_{\ell}$ is given by the representations $\rho_{\ell}: \Gamma_{F} \rightarrow G_{A}\left(\mathbb{Q}_{\ell}\right)$.

We closely follow the proof of [Noot 2009, Théorème 2.4]. Let the endomorphism algebra $L \subset \operatorname{End}^{0}(A)$ be as in Section 4.3. It is a product of number fields $L_{i}$ for $i=1, \ldots, s$. This decomposition gives rise to a decomposition up to isogeny $A \sim \prod_{i=1}^{s} A_{i}$ and to a corresponding decomposition $V=\bigoplus_{i=1}^{s} V_{i}$, where $V_{i}=\mathrm{H}_{1}\left(A_{i}(\mathbb{C}), \mathbb{Q}\right)$. For each $i$ one has $L_{i} \subset \operatorname{End}^{0}\left(A_{i}\right)$ and this action endows $V_{i}$ with the structure of $L_{i}$-vector space. It follows from Lemma 4.4 that each factor $A_{i}$ is either strictly tractable or that it is of type $D_{n}^{\mathbb{R}}$. If $A_{i}$ is not strictly tractable, it follows from the definition and again from Lemma 4.4 that there is another factor $A_{j}$ such that $L_{i} \cong L_{j}$ and the product $A_{i} \times A_{j}$ is strictly tractable. In this case we change definitions and put $A_{i}=A_{i} \times A_{j}$ and $L_{i}=L_{i} \times L_{j}$. We suppress the factors $A_{j}$ and $L_{j}$ and modify the value of $s$ accordingly. After these modifications, we have decompositions $L=\prod_{i=1}^{s} L_{i}$ and $A \sim \prod_{i=1}^{s} A_{i}$, where all factors $A_{i}$ are strictly tractable and each $L_{i}$ is either a field of a product $L_{i}^{\prime} \times L_{i}^{\prime}$ of fields.

Let $d_{i}=\operatorname{dim}_{L_{i}} V_{i}$ and let

$$
H=\prod_{i=1}^{s} \operatorname{Res}_{L_{i} / \mathbb{Q}} \mathrm{GL}_{/ L_{i}}\left(V_{i}\right)=\prod_{i=1}^{s} H_{i} \cong \prod_{i=1}^{s} \operatorname{Res}_{L_{i} / \mathbb{Q}} \mathrm{GL}_{d_{i} / L_{i}}
$$

be the centraliser of $L$ in $\operatorname{GL}(V)$. In the case where $L_{i} \cong L_{i}^{\prime} \times L_{i}^{\prime}$, the factor $H_{i}$ is

$$
\left(\operatorname{Res}_{L_{i}^{\prime} / \mathbb{Q}} \mathrm{GL}_{d_{i} / L_{i}^{\prime}}\right)^{2} \text {. }
$$


It is a linear algebraic group over $\mathbb{Q}$. The action of $G_{A}$ on $V$ commutes with the action of $\operatorname{End}^{0}(A)$, so $G_{A} \subset H$. The decomposition $H=\prod_{i=1}^{s} H_{i}$ corresponds to the decompositions of $L$, of $A$ and of $V$. In particular, $H_{i}$ is the only factor of $H$ acting nontrivially on $V_{i}$. Writing $G_{A_{i}}$ for the Mumford-Tate group of $A_{i}$ one has $G_{A_{i}} \subset H_{i}$.

Let $\Omega$ be an algebraically closed extension of $\mathbb{Q}$. For each $\mathbb{Q}$-algebra homomorphism $\iota: L \rightarrow \Omega$ there is a unique index $i=i(\iota)$ such that $\iota$ factors through $L \rightarrow L_{i} \rightarrow \Omega$, where $L \rightarrow L_{i}$ is the projection and $L_{i} \rightarrow \Omega$ a ring homomorphism. This final map is an embedding if $L_{i}$ is a field and an embedding of one of the factors of $L_{i}$ if $A_{i}$ is of type $D_{n}^{\mathbb{R}}$ and $L_{i}$ is a product $L_{i}^{\prime} \times L_{i}^{\prime}$. Let $d_{\iota}=d_{i}$. Extending the base field to $\Omega$ one obtains

$$
H_{/ \Omega} \cong \prod_{\iota: L \rightarrow \Omega} \mathrm{GL}_{d_{l} / \Omega}
$$

The group $G_{A / \Omega}$ embeds into this product and for $\iota: L \rightarrow \Omega$ we let $G_{\iota}$ be its image in the factor $\mathrm{GL}_{d_{\iota} / \Omega}$ corresponding to $\iota$.

This gives rise to similar decompositions of the Lie algebras. For $\mathfrak{h}=\operatorname{Lie}(H)$ we have $\mathfrak{h} \otimes_{\mathbb{Q}} \Omega=\bigoplus \mathfrak{h}_{\iota}=\bigoplus \operatorname{End}\left(V_{\iota}\right) \cong \bigoplus \mathfrak{g l}_{d_{\iota} / \Omega}$. The inclusion $G_{A} \subset H$ induces

$$
\mathfrak{g}_{A} \otimes \Omega=\operatorname{Lie}\left(G_{A}\right) \otimes \Omega \hookrightarrow \bigoplus_{\iota: L \rightarrow \Omega} \mathfrak{g}_{\iota} \subset \bigoplus_{\iota} \operatorname{End}\left(V_{\iota}\right)=\mathfrak{h} \otimes \Omega,
$$

where $\mathfrak{g}_{\iota} \subset \mathfrak{h}_{\iota}=\operatorname{End}\left(V_{\iota} \otimes \Omega\right)$ is the Lie algebra of $G_{\iota}$. For both the group $H_{/ \Omega} \cong \prod \mathrm{GL}_{d_{l} / \Omega}$ and the Lie algebra $\mathfrak{h} \otimes_{\mathbb{Q}} \Omega \cong \prod \mathfrak{g l}_{d_{l} / \Omega}$, the obvious action of $\sigma \in \operatorname{Aut}(\Omega)$ on the left hand side translates on the right hand side to

$$
\sigma:\left(x_{\iota}\right)_{\iota: L \rightarrow \Omega} \mapsto\left(\sigma\left(x_{\sigma^{-1} \iota}\right)\right)_{\iota: L \rightarrow \Omega}
$$

4.6. Monodromy and Frobenius weights. The $\ell$-adic realisations of $A$ decompose in the same way as the Betti realisation. Define $V_{i, \ell}=T_{\ell}\left(A_{i}\right) \otimes \mathbb{Q}_{\ell}$ for every $\ell$. The decomposition of $A$ gives rise to $\Gamma_{F_{v}}$-equivariant $L_{\ell}=L \otimes \mathbb{Q}_{\ell}$-linear decompositions $V_{\ell}=\bigoplus_{i=1}^{s} V_{i, \ell}$ and we have canonical $L_{i}$-linear isomorphisms $V_{i, \ell} \cong V_{i} \otimes_{\mathbb{Q}} \mathbb{Q}_{\ell}$.

Assume that $A$ has semistable reduction at $v$ and let further notation be as in Section 3.1. Let $q$ be the order of the residue field of $F_{v}$. To study the monodromy we follow Section 2.2, so we fix identifications $\mathbb{Q}_{\ell}(1) \cong \mathbb{Q}_{\ell}$ and define the monodromy operators $N_{\ell}^{\prime}$ by $(2.2 *)$. One has $N_{\ell}^{\prime} \in \mathfrak{g}_{A}^{\text {ss }} \otimes \mathbb{Q}_{\ell}$ by Section 3.2. Similarly, for each $A_{i}$ we have the monodromy operator $N_{i, \ell}^{\prime} \in \mathfrak{g}_{A_{i}}^{\mathrm{ss}} \otimes \mathbb{Q}_{\ell}$. Under the embedding $\mathfrak{g}_{A} \subset \bigoplus_{i=1}^{s} \mathfrak{g}_{A_{i}}$ the element $N_{\ell}^{\prime}$ maps to $\left(N_{i, \ell}^{\prime}\right)_{i=1, \ldots, s}$.

For $A$ and each one of the $A_{i}$, let the Frobenius weight cocharacters

$$
w_{\ell}: \mathbb{G}_{m / \overline{\mathbb{Q}}_{\ell}} \rightarrow G_{A / \overline{\mathbb{Q}}_{\ell}} \text { and } \quad w_{i, \ell}: \mathbb{G}_{m} / \overline{\mathbb{Q}}_{\ell} \rightarrow G_{A_{i} / \overline{\mathbb{Q}}_{\ell}}
$$


be as in Section 3.8. These cocharacters factor through the Mumford-Tate groups by Lemma 3.10. As in the case of the monodromy operators, the cocharacter

$$
\left(w_{i, \ell}\right)_{i=1, \ldots, s}: \mathbb{G}_{m / \overline{\mathbb{Q}}_{\ell}} \rightarrow \prod_{i=1, \ldots, s} G_{A_{i} / \overline{\mathbb{Q}}_{\ell}}
$$

is the composite of $w_{\ell}$ with the inclusion $G_{A} \subset \prod G_{A_{i}}$. It follows from Lemma 3.9 that $w_{\ell}$ and the $w_{i, \ell}$ split the monodromy filtrations on $V_{\ell}$ and on the $V_{i, \ell}$, respectively.

\section{Strictly tractable abelian varieties of types $A_{n}, C_{n}$ and $D_{n}^{\sharp 1}$}

5.1. We keep the notation of Section 3.1. In this section, we will assume that $A$ is a strictly tractable abelian variety and that its Mumford-Tate group $G_{A}$ is of type $A_{n}, C_{n}$ or $D_{n}^{\boxplus}$. According to Lemma 4.4, the algebra $L$ then is a field. In particular, where the group $H$ introduced in Section 4.5 is concerned, we have $d_{\iota}=d_{1}=d$ for all $\iota: L \rightarrow \Omega$. As in Section 4.6, consider the monodromy operator $N_{\ell}^{\prime}$ and the weight cocharacter $w_{\ell}$. It follows from Corollary 2.7 that the $H$-conjugacy class of $\left(N_{\ell}^{\prime}, w_{\ell}\right)$ is defined over $\mathbb{Q}$ and that it is independent of $\ell$, in accordance with the terminology developed in Section 2.3.

For any algebraically closed extension $\Omega$ of $\mathbb{Q}$, the Lie algebra $\mathfrak{g}^{\mathrm{ss}} \otimes \Omega$ is a direct sum of simple Lie algebras. The groups $G_{A}^{\mathrm{ad}}(\Omega)$ and $G_{A}^{\natural \text { ad }}(\Omega)$ act on each direct factor of this Lie algebra factor through a unique simple factor. Fix a factor $\mathfrak{g}_{l}^{\text {ss }}$ of $\mathfrak{g}^{\text {ss }} \otimes \Omega$ and consider the corresponding factors $G_{\iota}^{\text {der }}$ of $G_{A / \Omega}^{\text {der }}$ and $G_{\iota}^{\natural \text { ad }}$ of $G_{A / \Omega}^{\natural \text { ad }}$. Under the sequence of embeddings (4.5*), $\mathfrak{g}_{\iota}$ embeds into a simple factor $\mathfrak{h}_{\iota} \cong \mathfrak{g l}_{d}$ of $\mathfrak{h} \otimes \Omega$. If $G_{A}$ is of type $A_{n}$, then $\mathfrak{g}_{\iota}^{\text {ss }}$ and $G_{\iota}^{\text {der }}$ act on the direct factor $V_{\iota}$ of $V \otimes \Omega$ either as a multiple of the standard representation or as its dual. If $G_{A}$ is of type $C_{n}$ or $D_{n}^{\sharp}$ in the classification, $\mathfrak{g}_{\iota}^{\text {ss }}$ and $G_{\iota}^{\text {der }}$ act on $V_{\iota}$ as a multiple of the symplectic or the orthogonal representation respectively.

Let $\Omega \supset \mathbb{Q}_{\ell}$ be an algebraically closed field and let $\sigma \in \operatorname{Aut}(\Omega)$. As we saw above, $\left(N_{\ell}^{\prime}, w_{\ell}\right)$ and $\sigma\left(N_{\ell}^{\prime}, w_{\ell}\right) \in \mathfrak{h} \otimes \Omega$ are conjugate under the adjoint action of $H(\Omega)$. Writing $N_{\ell}^{\prime}=\left(N_{\ell, \ell}^{\prime}\right)_{\iota} \in \prod \mathfrak{h}_{\iota}$ as in $(4.5 *)$ and $w_{\ell}=\left(w_{\ell, \iota}\right)_{\iota}$ it follows from the formula $(4.5 \dagger)$ that the projections $\left(N_{\ell, l}^{\prime}, w_{\ell, \iota}\right)$ and $\sigma\left(N_{\ell, \sigma^{-1} \iota}^{\prime}, w_{\ell, \sigma^{-1} \iota}\right)$ of these pairs are $H_{l}(\Omega)$-conjugate.

In the case where $G_{A}$ is of type $A_{n}$, it trivially follows that $N_{\ell, \iota}^{\prime}$ and $\sigma\left(N_{\ell, \sigma^{-1}}^{\prime}\right)$ are conjugate under the action of $G_{l}^{\mathrm{ad}}(\Omega)=G_{l}^{\natural \mathrm{ad}}(\Omega)$ on $\mathfrak{g}_{l}$. In the cases where $G_{A}$ is of type $C_{n}$ or $D_{n}$, it follows from [Springer and Steinberg 1970, IV §2], in particular from 2.14, that $N_{\ell, \iota}^{\prime}$ and $\sigma\left(N_{\ell, \sigma^{-1} \iota}^{\prime}\right)$ are conjugate under the action of $G_{\iota}^{\natural \text { ad }}(\Omega)$ on $\mathfrak{g}_{\iota}$. See also [Humphreys $1995,7.11$ ] for a summary of the results concerning the nilpotent conjugacy classes in the classical Lie algebras.

Similarly, if $\Omega$ is an algebraically closed field containing $\mathbb{Q}_{\ell}$ and $\mathbb{Q}_{\ell^{\prime}}$ then the images of $N_{\ell}^{\prime}$ and $N_{\ell^{\prime}}^{\prime}$ in $\mathfrak{h}^{\text {ss }} \otimes \Omega$ are conjugate under $H(\Omega)$. The argument above 
implies that, for each $\iota$, the operators $N_{\ell, \iota}^{\prime}$ and $N_{\ell^{\prime}, \iota}^{\prime}$ are conjugate under the action of $G_{l}^{\natural \text { ad }}(\Omega)$ on $\mathfrak{g}_{\iota}$.

Next consider the weight cocharacter $w_{\ell}$. For each $\iota$, let $w_{\ell, \iota}$ be the projection of $w_{\ell}$ to $G_{\iota}$. Recall that by Lemma 3.9, the monodromy operator $N_{\ell, \iota}^{\prime}$ induces an isomorphism of the $t$-eigenspace of $w_{\ell, \iota}$ in $V_{\iota}$ onto the $t^{-1}$-eigenspace. Going through the arguments of [Springer and Steinberg 1970, IV], with $N_{\ell, \iota}^{\prime}$ playing the role of $X$, one deduces that there exists a basis of $V_{\iota}$ satisfying the conditions of [ibid., IV, 2.19(b)] such that $w_{\ell, \iota}$ is the inverse of the cocharacter $\lambda$ defined in [ibid., IV , 2.22]. In particular, $w_{\ell, \iota}$ factors through the derived group $G_{\iota}^{\text {der }}$. This fact can also quite easily be shown directly.

If $\Omega$ is an algebraically closed field and $\sigma \in \operatorname{Aut}(\Omega)$ then, for $X=\sigma\left(N_{\ell, \sigma^{-1} \ell}^{\prime}\right)$, there is a basis of $V_{\iota}$ as in [ibid., IV, 2.19(b)] such that $\sigma\left(w_{\ell, \sigma^{-1}}\right)$ coincides with $\sigma\left(\lambda^{-1}\right)$. We know that $N_{\ell, \iota}^{\prime}$ and $\sigma\left(N_{\ell, \sigma^{-1}}^{\prime}\right)$ are conjugate under $G_{l}^{\natural \text { ad }}(\Omega)$. Moreover, any two bases of $V$ that satisfy the conditions of [ibid., IV, 2.19(b)] are conjugate under the centraliser $Z_{\ell, \iota}^{\natural \text { ad }}$ of $N_{\ell, \iota}^{\prime}$ in $G_{l}^{\natural \text { ad }}$. It follows that $\left(N_{\ell, l}^{\prime}, w_{\ell, l}\right)$ and $\sigma\left(N_{\ell, \sigma^{-1} \iota}^{\prime}, w_{\ell, \sigma^{-1} \iota}\right)$ are $G_{\iota}^{\natural \text { ad }}(\Omega)$-conjugate.

If $\ell$ and $\ell^{\prime}$ are two prime numbers and if $\Omega$ is an algebraically closed field containing both $\mathbb{Q}_{\ell}$ and $\mathbb{Q}_{\ell^{\prime}}$, then the same argument, applied to $\left(N_{\ell, \ell}^{\prime}, w_{\ell, \ell}\right)$ and $\left(N_{\ell^{\prime}, l}^{\prime}, w_{\ell^{\prime}, l}\right)$, proves that these two pairs are $G_{l}^{\natural \text { ad }}(\Omega)$-conjugate.

Proposition 5.2. Let notation and assumptions be as above. In particular, $A / F$ is a strictly tractable abelian variety of type $A_{n}, B_{n}$ or $D_{n}^{\sharp !}$.

- For every $\ell$, every algebraically closed field $\Omega \supset \mathbb{Q}_{\ell}$ and every $\sigma \in \operatorname{Aut}(\Omega)$, the image of $\left(N_{\ell}^{\prime}, w_{\ell}\right)$ in $\mathfrak{g} \otimes \Omega \times X\left(G_{A / \Omega}\right)$ is conjugate to $\sigma\left(N_{\ell}^{\prime}, w_{\ell}\right)$ under the adjoint action of $G_{A}^{\natural \text { ad }}(\Omega)$.

- If $\Omega$ is an algebraically closed field containing both $\mathbb{Q}_{\ell}$ and $\mathbb{Q}_{\ell^{\prime}}$, then the images in $\mathfrak{g} \otimes \Omega \times X\left(G_{A / \Omega}\right)$ of $\left(N_{\ell}^{\prime}, w_{\ell}\right)$ and $\left(N_{\ell^{\prime}}^{\prime}, w_{\ell^{\prime}}\right)$ are $G_{A}^{\natural \text { ad }}(\Omega)$-conjugate.

Proof. As $A$ is tractable, the group $G_{A / \Omega}^{\text {der }}$ is the product of its almost simple factors. If $G_{A}$ is of type $C_{n}$ or $D_{n}^{\mathbb{}}$, then $G_{A / \Omega}^{\mathrm{der}}$ is the product of the $G_{l}^{\mathrm{der}}$. In the case where $G_{A}$ is of type $A_{n}$, we saw in the proof of Lemma 4.4 that the complex conjugation acts nontrivially on each component of the Dynkin diagram. If $n \geq 2$, it follows from [Noot 2006, 5.1] that $L$ is a CM field and hence that the complex conjugation defines an nontrivial involution $\iota \mapsto \iota^{\prime}$ on the set of embeddings $L \rightarrow \Omega$. In this case, $G_{A / \Omega}^{\mathrm{der}}$ is a product of groups $\Delta_{\left\{l, l^{\prime}\right\}}$, where each $\Delta_{\left\{l, \iota^{\prime}\right\}} \subset G_{\iota}^{\mathrm{der}} \times G_{\iota^{\prime}}^{\mathrm{der}}$ is the graph of an isomorphism $G_{l}^{\mathrm{der}} \cong G_{\iota^{\prime}}^{\mathrm{der}}$. Identifying $\Delta_{\left\{l, l^{\prime}\right\}}$ with $G_{l}^{\mathrm{der}}$ through the projection on the first factor, the representation of $\Delta_{\left\{l, l^{\prime}\right\}}$ on $V_{\iota}$ is a multiple of the representation with highest weight $\varpi_{1}$ and $V_{\iota^{\prime}}$ is its dual, a multiple of the representation with highest weight $\varpi_{n}$. The case where $n=1$ is left to the reader.

The $N_{\ell, \iota}^{\prime}$ belong to $\mathfrak{g}_{\iota}^{\text {ss }}$ and, as we pointed out in Section 5.1, the $w_{\ell, \iota}$ factor through $G_{l}^{\text {der }}$. The proposition follows from the fact, proved in Section 5.1, that 
$\left(N_{\ell, \ell}^{\prime}, w_{\ell, \iota}\right)$ and $\sigma\left(N_{\ell, \ell}^{\prime}, w_{\ell, \ell}\right)$ are $G_{\iota}^{\natural \text { ad }}(\Omega)$-conjugate for each $\iota$, combined with the formula $(4.5 \dagger)$.

5.3. The centraliser of $\left(N_{\ell}^{\prime}, w_{\ell}\right)$ in $G_{A / \bar{Q}_{\ell}}^{\natural}$ We return to the construction 3.3 of the group $G_{A}^{\natural \text { ad }}$. In the case considered here, this group is the adjoint group of a $\overline{\mathbb{Q}}$-group $G_{A}^{\natural}$ containing the derived Mumford-Tate group $G_{A / \overline{\mathbb{Q}}}^{\mathrm{der}}$. In fact, one has to take $G_{A}^{\natural}=G_{A / \bar{Q}}^{\mathrm{der}}$ if $G_{A}$ is of type $A_{n}$ or $C_{n}$ and in this case we put $G_{\iota}^{\natural}=G_{\iota}^{\mathrm{der}}$ for each embedding $\iota: L \rightarrow \overline{\mathbb{Q}}$. If $G_{A}$ is of type $D_{n}^{\mathbb{W}}$, then

$$
G_{A / \overline{\mathbb{Q}}}^{\mathrm{der}}=\prod G_{\iota}^{\mathrm{der}},
$$

where each $G_{l}^{\text {der }} \cong \mathrm{SO}_{2 n}$. We put $G_{\imath}^{\natural}=\mathrm{O}_{2 n}$ and $G_{A}^{\natural}=\prod G_{l}^{\natural}$. In all cases, it is clear from the construction Section 3.3 that $G_{A / \bar{Q}}^{\text {der }} \subset G_{A}^{\natural}$ is the identity component and that the group $G_{A}^{\natural \text { ad }}$ defined in that construction is indeed the adjoint group of $G_{A}^{\natural}$. Working with this group $G_{A}^{\natural}$, we may apply [Springer and Steinberg 1970, IV].

In what follows, the centralisers $C_{\ell}^{\natural} \subset G_{A / \overline{\mathbb{Q}}_{\ell}}^{\natural}$ and $C_{\ell} \subset G_{A / \overline{\mathbb{Q}}_{\ell}}$ of the pair $\left(N_{\ell}^{\prime}, w_{\ell}\right)$ will play an important role. The group $C_{\ell}$ is the subgroup of $G_{A / \overline{\mathbb{Q}}_{\ell}}$ generated by $C_{\ell}^{\natural} \cap G_{A / \overline{\mathbb{Q}}_{\ell}}^{\text {der }}$ and the centre of $G_{A / \overline{\mathbb{Q}}_{\ell}}$. The embedding

$$
G_{A / \overline{\mathbb{Q}}_{\ell}}^{\natural} \rightarrow \prod_{\ell} G_{l / \overline{\mathbb{Q}}_{\ell}}^{\natural}
$$

gives rise to a similar embedding $C_{\ell / \overline{\mathbb{Q}}_{\ell}}^{\natural} \rightarrow \prod_{\iota} C_{\ell, l}^{\natural}$, where each $C_{\ell, \iota}^{\natural}$ is the centraliser of $\left(N_{\ell, \iota}^{\prime}, w_{\ell, \iota}\right)$ in $G_{\iota}^{\natural}$. We first determine these groups $C^{\natural} \ell, \iota$.

By Lemma 3.9, $N_{\ell}^{\prime}$ induces an isomorphism from the $t$-eigenspace of $w_{\ell}$ onto the $t^{-1}$-eigenspace of $w_{\ell}$. As we saw in Section 5.1, this implies that, taking $G=G_{\iota}^{\natural}$ and $X=N_{\ell, \iota}^{\prime}$ in [Springer and Steinberg 1970, IV §2], the proper choice of a basis of $V_{\iota}$ ensures that the cocharacter $w_{\ell, \iota}$ is the inverse of the cocharacter $\lambda$ defined in that paper, IV 2.22. The group $C_{\ell, \iota}^{\natural}$ is therefore equal to the group $C$ of [ibid., IV 2.23(iii)]. Note that, contrary to what is affirmed in that statement, this group is not necessarily connected. Indeed, there may be two connected components; see [ibid., 2.25 and 2.26].

To give an explicit description of $C_{\ell, l}^{\natural}$, let the 1-motive $M^{\prime}$ and the dimensions $r=r^{\star}$ and $g$ be as in Section 3.8. In this case, $r=r^{\star}$ is equal to the dimension of both the $t$ and the $t^{-1}$-eigenspaces of $w_{\ell}$. Since $N_{\ell, \iota}^{\prime}$ is nilpotent of echelon at most 2 , it is easily deduced from [ibid., IV 1.8, 2.25] that the group $C_{\ell, \ell}^{\natural}$ is isomorphic to the $\overline{\mathbb{Q}}_{\ell}$-group

- $\mathrm{SL}_{r} \times \mathrm{SL}_{2 g}$ if $G_{A}$ is of type $A_{n}$,

- $\mathrm{O}_{r} \times \mathrm{Sp}_{2 g}$ if $G_{A}$ is of type $C_{n}$ or

- $\mathrm{Sp}_{r} \times \mathrm{O}_{2 g}$ if $G_{A}$ is of type $D_{n}$. 
Each factor $C_{\ell, \ell}^{\natural}$ is therefore a product $C_{\ell, \ell, 0}^{\natural} \times C_{\ell, \ell,-1}^{\natural}$ and this decomposition is determined by the cocharacter $w_{\ell, \iota}$. In fact, for each integer $k$ let $V_{\ell}^{k-1}$ and $V_{\ell, l}^{k-1}$ be the $t^{k}$-eigenspaces of $w_{\ell}$ and $w_{\ell, \iota}$ in $V_{\ell}=V \otimes \mathbb{Q}_{\ell}$ and $V_{\iota} \otimes \overline{\mathbb{Q}}_{\ell}$, respectively. This seemingly confusing notation is consistent with Section 1.10. As $C_{\ell, \iota}^{\natural}$ commutes with $w_{\ell, \iota}$, it respects the grading $V_{\ell, \iota}=\bigoplus_{k=-2,-1,0} V_{\ell, \iota}^{k}$ and it follows from [ibid., IV 1.8, 2.25] that for $k=0,-1$, the group $C_{\ell, \iota, k}^{\natural}$ is the image of $C_{\ell}^{\natural}$ in $\operatorname{GL}\left(V_{\ell, \ell}^{k}\right)$. For a group of type $A_{n}$ or $C_{n}$, this embedding is given by the standard or symplectic representation, respectively, and for a group of type $B_{n}$ or $D_{n}$ it is defined by the orthogonal representation. The monodromy operator $N_{\ell, l}$ defines an isomorphism of the representations of $C_{\ell, l, 0}^{\natural}$ on $V_{\ell, \iota}^{0}$ and on $V_{\ell, l}^{-2}$.

The decomposition above can be defined on the level of the group $C_{\ell}$ by taking $C_{\ell, k}$ equal to the image of $C_{\ell}$ in $\operatorname{GL}\left(V_{\ell}^{k}\right)$, for $k=0,-1$. Finally, let $C_{\ell, \ell, k}$ be the image of $C_{\ell / \overline{\mathbb{Q}}_{\ell}}$ in $\operatorname{GL}\left(V_{\ell, \ell}^{k}\right)$, so that each $C_{\ell, \ell, k}^{\mathrm{der}} \subset C_{\ell, \ell, k}^{\natural}$ is the identity component, with equality for all factors other than those isomorphic to an $\mathrm{SO}_{2 n}$.

5.4. The representations $\rho_{\ell}^{\prime}$. We now turn to the representations $\rho_{\ell}^{\prime}$ of the Weil group $W_{v}=W_{F_{v}}$. For general $\psi \in W_{v}$, the image $\rho_{\ell}^{\prime}(\psi)$ does not belong to $C_{\ell}\left(\mathbb{Q}_{\ell}\right)$ so in order to apply the arguments of [Noot 2009, §2], we replace $C_{\ell}$ by the group $\widetilde{C}_{\ell} \subset G_{A / \bar{Q}_{\ell}}$ generated by $C_{\ell}$ and the image of $w_{\ell}$. For $\psi \in W_{v}$ one has

$$
\operatorname{Ad}\left(\rho_{\ell}^{\prime}(\psi)\right)\left(N_{\ell}^{\prime}\right)=q^{\alpha(\psi)} N_{\ell}^{\prime}=\operatorname{Ad}\left(w_{\ell}\left(q^{\alpha(\psi) / 2}\right)\right)\left(N_{\ell}^{\prime}\right) .
$$

On the other had, it follows from Lemma 2.5 that $\rho_{\ell}^{\prime}(\psi)$ and $w_{\ell}$ commute. This implies that $\rho_{\ell}^{\prime}(\psi) w_{\ell}\left(q^{-\alpha(\psi) / 2}\right)$ lies in $C_{\ell}\left(\overline{\mathbb{Q}}_{\ell}\right)$ and hence that $\rho_{\ell}^{\prime}(\psi) \in \widetilde{C}_{\ell}\left(\mathbb{Q}_{\ell}\right)$. The action of $\widetilde{C}_{\ell}$ on $V_{\ell}$ respects the grading $V_{\ell}=\bigoplus_{k=-2,-1,0} V_{\ell}^{k}$ so, for $k=0,-1$, it makes sense to define $\widetilde{C}_{\ell, k}$ as the image of $\widetilde{C}_{\ell}$ in $\operatorname{GL}\left(V_{\ell, \ell}^{k}\right)$. The adjoint action of $C_{\ell}^{\natural}$ on $C_{\ell}$ extends to an action of $C_{\ell}^{\natural}$ on $\widetilde{C}_{\ell}$, with the former group acting trivially on the image of $w_{\ell}$.

Recall that, according to Lemma 4.4, the algebra $L$ is a field in the cases considered here. We write $\mathbb{A}_{(L)}^{d}=\operatorname{Res}_{L / \mathbb{Q}} \mathbb{A}_{/ L}^{d}$. The discussion above shows that taking the $L_{\ell}$-linear characteristic polynomials of the elements of $\widetilde{C}_{\ell}$ acting on the $w_{\ell}$-eigenspace $V_{\ell}^{-1}$, one defines a map

$$
P_{L}^{\prime}: \widetilde{C}_{\ell} \rightarrow \widetilde{C}_{\ell,-1} \rightarrow \mathbb{A}_{(L) / \overline{\mathbb{Q}}_{\ell}}^{2 g}
$$

We will also write $P_{L}^{\prime}$ for the map $\widetilde{C}_{\ell,-1} \rightarrow \mathbb{A}_{(L) / \bar{Q}_{\ell}}^{2 g}$. As in [Noot 2009], the maps $P_{L}^{\prime}$ factor through the quotients of $\widetilde{C}_{\ell}$ and $\widetilde{C}_{\ell,-1}$ by the adjoint $C_{\ell}^{\natural}$-action.

It follows from Proposition 1.8 and from Section 3.8 that for any $\psi \in W_{v}$, the characteristic polynomial of $\rho_{\ell}^{\prime}(\psi)$ acting as an $L_{\ell}$-linear automorphism on $V_{\ell}^{-1}$ has coefficients in $L$ and is independent of $\ell$. This proves the following lemma.

Lemma 5.5. Under the hypotheses above, the image of $\rho_{\ell}^{\prime}(\psi) \in \widetilde{C}_{\ell}\left(\mathbb{Q}_{\ell}\right)$ under the map $P_{L}^{\prime}$ defined in $(5.4 *)$ lies in $\mathbb{A}_{(L)}^{2 g}(\mathbb{Q})$ and is independent of $\ell$. 
5.6. Remark. The statement of the lemma also holds for the image of $\rho_{\ell}^{\prime}(\psi)$ under the map $P_{L}^{\prime \prime}: \widetilde{C}_{\ell} \rightarrow \mathbb{A}^{r}(L)$ defined by taking the characteristic polynomial on $V_{\ell}^{0}$. This observation is of little interest for weakly neat elements.

Proposition 5.7. The main theorem, Theorem 3.6, holds if $A$ is strictly tractable and its Mumford-Tate group $G_{A}$ is of type $A_{n}, C_{n}$ or $D_{n}^{\mathbb{Q}}$.

Proof. As the assumptions of Theorem 3.6 are now in force, $A$ has semistable reduction at $v$ and the image $\rho_{\ell}^{\prime}\left(\Phi_{v}\right)$ of Frobenius is weakly neat. This implies that the restriction of $\rho_{\ell}^{\prime}$ to $I_{\bar{b}}$ is trivial and that $\rho_{\ell}^{\prime}\left(\Phi_{v}\right)$ acts on $V_{\ell}^{0}$ as multiplication by $\varepsilon= \pm 1$ and on $V_{\ell}^{-2}$ as multiplication by $q \varepsilon$.

As in Section 5.1, let $\Omega \supset \mathbb{Q}_{\ell}$ be an algebraically closed field and let $\sigma \in \operatorname{Aut}(\Omega)$. We have to show that the pairs $\left(N_{\ell}^{\prime}, \rho_{\ell}^{\prime}\left(\Phi_{v}\right)\right)$ and $\left(\sigma\left(N_{\ell}^{\prime}\right), \sigma\left(\rho_{\ell}^{\prime}\left(\Phi_{v}\right)\right)\right)$ are conjugate under the action of $G_{A}^{\natural}(\Omega)$. By Proposition 5.2, there is a $g \in G_{A}^{\natural}(\Omega)$ such that

$$
\left(N_{\ell}^{\prime}, w_{\ell}\right)=\operatorname{Ad}(g)\left(\sigma\left(N_{\ell}^{\prime}\right), \sigma\left(w_{\ell}\right)\right) .
$$

This implies that $\rho_{\ell}^{\prime}\left(\Phi_{v}\right)$ and $g \sigma\left(\rho_{\ell}^{\prime}\left(\Phi_{v}\right)\right) g^{-1}$ belong to $\widetilde{C}_{\ell}(\Omega)$. As $C_{\ell}^{\natural}$ centralises $\left(N_{\ell}^{\prime}, w_{\ell}\right)$, it is enough to show that $\rho_{\ell}^{\prime}\left(\Phi_{v}\right)$ and $g \sigma\left(\rho_{\ell}^{\prime}\left(\Phi_{v}\right)\right) g^{-1}$ are conjugate under $C_{\ell}^{\natural}(\Omega)$. By Section 1.9 and the proof of Proposition 2.6, the element $\rho_{\ell}^{\prime}\left(\Phi_{v}\right)$ is semisimple. Moreover

$$
P_{L}^{\prime}\left(g \sigma\left(\rho_{\ell}^{\prime}\left(\Phi_{v}\right)\right) g^{-1}\right)=\sigma\left(P_{L}^{\prime}\left(\rho_{\ell}^{\prime}\left(\Phi_{v}\right)\right)\right)=P_{L}^{\prime}\left(\rho_{\ell}^{\prime}\left(\Phi_{v}\right)\right),
$$

where the former equality is elementary and the latter one follows from Lemma 5.5. The projections of these elements to $\widetilde{C}_{\ell,-1}(\Omega)$ are semisimple and weakly neat and their projections to $C_{\ell, 0}(\Omega)$ lie in the centre of this group. The required statement therefore follows from Lemma 5.8.

For the $\ell$-independence, let $\ell$ and $\ell^{\prime}$ be two prime numbers and let $\Omega$ be an algebraically closed field containing $\mathbb{Q}_{\ell}$ and $\mathbb{Q}_{\ell^{\prime}}$. It follows from Proposition 5.2 that there exists $g \in G_{A}^{\natural}(\Omega)$ such that $\left(N_{\ell}^{\prime}, w_{\ell}\right)=\operatorname{Ad}(g)\left(N_{\ell^{\prime}}^{\prime}, w_{\ell^{\prime}}\right)$. Exactly as before we combine the Lemmas 5.5 and 5.8 to show that $\rho_{\ell}^{\prime}\left(\Phi_{v}\right)$ and $\rho_{\ell^{\prime}}^{\prime}\left(\Phi_{v}\right)$ are conjugate under $C_{\ell}^{\natural}(\Omega)$.

Lemma 5.8. Let $\widetilde{C}_{\ell}, C_{\ell}^{\natural}$ and $P_{L}^{\prime}$ be as above and let $g_{1}, g_{2} \in \widetilde{C}_{\ell}(\Omega)$ be semisimple elements whose projections to $C_{\ell,-1}^{\natural}(\Omega)$ are weakly neat and whose projections to $\widetilde{C}_{\ell, 0}(\Omega)$ act on $V_{\ell}^{0}$ by the same scalar multiplication. If $P_{L}^{\prime}\left(g_{1}\right)=P_{L}^{\prime}\left(g_{2}\right)$ then $g_{1}$ and $g_{2}$ are conjugate under $C_{\ell}^{\natural}(\Omega)$.

Proof. This essentially results from [Noot 2009, Lemmas 2.5 and 2.6]. First note that the variety $\operatorname{Conj}^{\prime}\left(\widetilde{C}_{\ell}\right)$ considered in [ibid., Lemma 2.5 ] is the variety of semisimple $C_{\ell}^{\natural}$-conjugacy classes in $\widetilde{C}_{\ell}$. Similarly, for each $\iota$ and $k$, the variety $\operatorname{Conj}^{\prime}\left(\widetilde{C}_{\ell, l, k}\right)$ is the variety of semisimple $C_{\ell, l, k}^{\natural}$-conjugacy classes in $\widetilde{C}_{\ell, \ell, k}$. It follows that $\operatorname{Conj}^{\prime}\left(\widetilde{C}_{\ell}\right)(\Omega)$ and $\operatorname{Conj}^{\prime}\left(\widetilde{C}_{\ell, \ell, k}\right)(\Omega)$ are the sets of semisimple $C_{\ell}^{\natural}(\Omega)$ and $C_{\ell, \ell, k}^{\natural}(\Omega)$-conjugacy classes in $\widetilde{C}_{\ell}(\Omega)$ and in $\widetilde{C}_{\ell, \ell, k}(\Omega)$, respectively. 
It thus follows from [ibid., Lemma 2.5] and its proof that the map

$$
\widetilde{C}_{\ell}(\Omega) \rightarrow \widetilde{C}_{\ell, 0}(\Omega) \times \widetilde{C}_{\ell,-1}(\Omega) \rightarrow \prod_{\ell} \widetilde{C}_{\ell, l, 0}(\Omega) \times \prod_{\ell} \widetilde{C}_{\ell, l,-1}(\Omega)
$$

induces an injection on weakly neat $C_{\ell}^{\natural}(\Omega)$-conjugacy classes. As the images of $g_{1}$ and $g_{2}$ in $\widetilde{C}_{\ell, 0}(\Omega)$ are equal and since these images are $C_{\ell}^{\natural}(\Omega)$-invariant, it is sufficient to show that the images of $g_{1}$ and $g_{2}$ in each $\operatorname{Conj}^{\prime}\left(\widetilde{C}_{\ell, l,-1}\right)(\Omega)$ coincide.

To this end, note that each map

$$
P_{L}^{\prime}: \operatorname{Conj}^{\prime}\left(\widetilde{C}_{\ell, l,-1}\right)(\Omega) \rightarrow \mathbb{A}^{2 g}(\Omega)
$$

is injective. This is the statement of [ibid., Lemma 2.6] for the group $C_{\ell, L,-1}^{\text {der }}$, with $\Omega$ instead of $\overline{\mathbb{Q}}$. Since $C_{\ell, l,-1}^{\mathrm{der}}$ is of type $A_{n}, C_{n}$ or $D_{n}^{\mathbb{\sharp}}$, it follows from the remark in the beginning of the proof of [ibid., Lemma 2.6] that the lemma in question is valid in this setting.

\section{Strictly tractable abelian varieties of types $B_{n}$ and $D_{n}^{\mathbb{R}}$}

6.1. The monodromy in a Mumford-Tate group of type $B_{n}$. With the notation of Section 3.1, we turn to the case where $A / F$ is a strictly tractable abelian variety with Mumford-Tate group $G_{A}$ of type $B_{n}$. We will adapt the arguments of Section 5 to this case. The endomorphism algebra $L$ and the group $H$ are defined as in Sections 4.3 and 4.5. As in the previous cases, it follows from Lemma 4.4 that $L$ is a field. Moreover we have $G_{A}^{\natural \text { ad }}=G_{A}^{\text {ad }}$. For each prime number $\ell$, the monodromy operator $N_{\ell}^{\prime}$ and the Frobenius weight cocharacter $w_{\ell}$ are defined as before. Each $w_{\ell}$ acts on $V_{\ell}=V \otimes \mathbb{Q}_{\ell}$ with at most three eigenvalues. It presents a single weight if and only if $A$ has good reduction, which is the case if and only if $N_{\ell}^{\prime}=0$.

Recall the decomposition $L \otimes \overline{\mathbb{Q}}=\bigoplus_{\iota} \overline{\mathbb{Q}}$ from Section 4.3, where the direct sum is indexed by the maps $\iota: L \rightarrow \overline{\mathbb{Q}}$. As in Sections 4.3 and 4.5, we consider the resulting decompositions $V \otimes \overline{\mathbb{Q}}=\bigoplus_{\iota} V_{\iota}$ and $\mathfrak{h} \otimes \overline{\mathbb{Q}}=\bigoplus_{\iota} \mathfrak{h}_{\iota}$ as well as the embedding $\mathfrak{g} \otimes \overline{\mathbb{Q}} \hookrightarrow \bigoplus_{\iota} \mathfrak{g}_{\iota}$. We write $G_{\iota}$ for the image of $G_{A / \overline{\mathbb{Q}}}$ in $\operatorname{GL}\left(V_{l}\right)$. The derived group $G_{A / \bar{Q}}^{\text {der }}$ identifies with the product $\prod_{\iota} G_{\iota}^{\text {der }}$. Each $G_{\iota}^{\text {der }}$ is a spin group of type $B_{n}$ and its representation on $V_{\iota}$ is a multiple of the irreducible representation $V_{\iota}^{\text {irr }}$ of highest weight $\varpi_{n}$ and hence of dimension $d=2^{n}$.

As before, let $\left(N_{\ell, \imath}^{\prime}\right)_{\iota}$ be the image of $N_{\ell}^{\prime}$ in $\bigoplus_{\iota} \mathfrak{g}_{\iota} \otimes \overline{\mathbb{Q}}_{\ell}$. Of course, $N_{\ell}^{\prime}$ lies in $\mathfrak{g}^{\mathrm{ss}} \otimes \mathbb{Q}_{\ell}$ and $N_{\ell, \ell}^{\prime} \in \mathfrak{g}_{l}^{\text {ss }} \otimes \overline{\mathbb{Q}}_{\ell}$. The operator $N_{\ell}^{\prime}$ is $L_{\ell}$-linear so it belongs to $\mathfrak{h} \otimes \mathbb{Q}_{\ell}$ and it follows from the Corollary 2.7 that its $H\left(\overline{\mathbb{Q}}_{\ell}\right)$-orbit is defined over $\mathbb{Q}$. This implies the rank of the projection $N_{\ell, \iota}^{\prime}$ is independent of $\iota$. If $N_{\ell, \iota}^{\prime}=0$ for some $\iota$, then all $N_{\ell, \iota}^{\prime}$ are trivial and then the abelian variety $A$ has potentially good reduction. This is the situation treated in [Noot 2009]. In what follows, we will assume that this is not the case. We investigate the possible ranks of the $N_{\ell, \iota}^{\prime}$ and the corresponding forms of the cocharacters $w_{\ell, \iota}$. 
Let $T_{\iota}$ be a maximal torus of $G_{\iota}$ and let $\widetilde{T}_{\iota}=\mathbb{G}_{m}^{d}$ be a maximal torus of $\operatorname{GL}\left(V_{\iota}^{\mathrm{irr}}\right)$ containing it. According to [ibid., 2.6.4] we have $d=2^{n}$ and we can assume that $T_{\iota}$ is the image of the application $\mathbb{G}_{m}^{n+1} \rightarrow \widetilde{T}_{\iota}$ given by

$$
\left(\lambda_{0}, \lambda_{1}, \ldots, \lambda_{n}\right) \mapsto\left(\lambda_{0} \lambda_{1}^{\varepsilon_{1}} \cdots \lambda_{n}^{\varepsilon_{n}}\right)_{\left(\varepsilon_{1}, \ldots, \varepsilon_{n}\right)=( \pm 1, \ldots, \pm 1)},
$$

where the factors of $\widetilde{T}_{\iota}$ are indexed by $n$-tuples of signs $\left(\varepsilon_{1}, \ldots, \varepsilon_{n}\right)$.

For each $\iota$, the Frobenius weight cocharacter $w_{\ell}$ defines a cocharacter $w_{\ell, \iota}$ of $G_{\iota} / \overline{\mathbb{Q}}_{\ell}$. The monodromy operator $N_{\ell, \iota}^{\prime}$ defines an isomorphism between the $t$ and $t^{-1}$ eigenspaces of $w_{\ell, \iota}$ acting on $V_{\iota}$, so these eigenspaces have the same dimension and it follows that $w_{\ell, \iota}$ factors through $G_{\iota / \overline{\mathbb{Q}}_{\ell}}^{\mathrm{der}}$. Up to conjugation by an element of $G_{\iota}^{\text {der }}\left(\overline{\mathbb{Q}}_{\ell}\right)$, we can assume that $w_{\ell, \iota}$ factors through $T_{\iota} / \overline{\mathbb{Q}}_{\ell}$. It then lifts to a quasicocharacter $\tilde{w}_{\ell, \iota}$ with values in $\mathbb{G}_{m}^{n+1}$. The filtration on $V_{\iota}^{\text {irr }}$ defined by $w_{\ell, \iota}$ has at most three weights and it follows that $\tilde{w}_{\ell, \iota}$ projects nontrivially to at most two factors of $\mathbb{G}_{m}^{n}$. Moreover, if it projects nontrivially to two factors, then the two projections must coincide.

The filtration by Frobenius weights coincides with the monodromy filtration and it follows that

- if $\tilde{w}_{\ell, \iota}$ is trivial, then $N_{\ell, \iota}^{\prime}=0$;

- if $\tilde{w}_{\ell, \iota}$ projects nontrivially to exactly one factor of $\mathbb{G}_{m}^{n}$, then $N_{\ell, \iota}^{\prime}$ is of rank $2^{n-1}$ (as an endomorphism of $V_{\iota}^{\text {irr }}$ ); and

- if $\tilde{w}_{\ell, \iota}$ projects nontrivially to exactly two factors of $\mathbb{G}_{m}^{n}$, then $N_{\ell, \iota}^{\prime}$ is of rank $2^{n-2}$.

The first possibility is excluded by the hypothesis that $N_{\ell}^{\prime} \neq 0$.

As for the other cases, we consider the adjoint group $G_{\iota}^{\text {ad }}$ of $G_{\iota}^{\text {der }}$, which is isomorphic to the special orthogonal group $\mathrm{SO}_{2 n+1 / \overline{\mathbb{Q}}_{\ell}}$. Let $W_{\iota}$ be the orthogonal representation of this group, that is, the representation with highest weight $\varpi_{1}$, and let $w_{\ell, \iota}^{\mathrm{ad}}$ be the projection of the cocharacter $w_{\ell, \iota}$ to $G_{\iota}^{\mathrm{ad}}$.

If we are in the second case then $w_{\ell, \iota}^{\text {ad }}$ acts on $W_{\iota}$ with eigenvalues $t, 1$ and $t^{-1}$ and the eigenspaces for $t$ and for $t^{-1}$ are 1-dimensional. The relation

$$
\operatorname{Ad}\left(w_{\ell, \iota}(t)\right)\left(N_{\ell, \iota}^{\prime}\right)=t^{2} N_{\ell, \iota}^{\prime}
$$

implies that the same relation holds with $w_{\ell, l}^{\text {ad }}$ instead of $w_{\ell, l}$. It follows that the Jordan normal form of the image of $N_{\ell, \iota}^{\prime}$ in the orthogonal representation of $\mathfrak{g}_{l}^{\text {ss }}$ has one block of size 2 and that all other blocks are of size 1. This is impossible according to [Springer and Steinberg 1970, IV 2.14; Humphreys 1995, 7.11] so the second possibility is excluded.

We study of the conjugacy class of $\left(N_{\ell}^{\prime}, w_{\ell}\right)$ in the third, and only possible, case. The argument above shows that the image of $N_{\ell, \ell}^{\prime}$ in the orthogonal representation 
$W_{\iota}$ of $\mathfrak{g}_{\iota}^{\text {ss }}$ has two blocks of size 2 and that all other blocks are of size 1 . Considering the orthogonal representation $W_{\iota}$, we also see that if we take $X=N_{\ell, \iota}^{\prime}$ in [Springer and Steinberg 1970, IV 2.19(b)], then we can assume that $w_{\ell, l}^{\mathrm{ad}}$ is the inverse of the cocharacter $\lambda$ of [ibid., IV 2.22]. This remains true after passing to any algebraically closed field $\Omega \supset \overline{\mathbb{Q}}_{\ell}$ and also after replacing the data $\left(N_{\ell}^{\prime}, w_{\ell}\right)$ by $\sigma\left(N_{\ell}^{\prime}, w_{\ell}\right)$, where $\sigma$ is an automorphism of $\Omega$. Applying [ibid., IV, §2] in the same way as in Section 5.1, we prove that for any such $\Omega$ and $\sigma$, the pairs $\left(N_{\ell, \ell}^{\prime}, w_{\ell, l}^{\text {ad }}\right)$ and $\sigma\left(N_{\ell, l}^{\prime}, w_{\ell, l}^{\mathrm{ad}}\right)$ are conjugate under the $G_{\iota}^{\mathrm{der}}(\Omega)$-action. It follows that this is also the case for $\left(N_{\ell, l}^{\prime}, w_{\ell, l}\right)$ and $\sigma\left(N_{\ell, \ell}^{\prime}, w_{\ell, \ell}\right)$.

One shows by the same argument that if $\ell^{\prime}$ is a second prime number and if $\Omega$ is an algebraically closed field containing $\mathbb{Q}_{\ell}$ and $\mathbb{Q}_{\ell^{\prime}}$, then $\left(N_{\ell}^{\prime}, w_{\ell}\right)$ and $\left(N_{\ell^{\prime}}^{\prime}, w_{\ell^{\prime}}\right)$ are $G_{A}(\Omega)$-conjugate. This proves Proposition 5.2 in the case where $A / F$ is a strictly tractable abelian variety of type $B_{n}$.

\subsection{The Frobenius elements in Mumford-Tate groups of type $B_{n}$. To prove the} conjugacy of the Frobenius elements, we adapt the argument used from Section 5.3 to Lemma 5.8. On the one hand, notation is simplified because $G_{A}^{\natural a d}=G_{A}^{\text {ad }}$, but on the other hand, they are complicated by the fact that we need to consider the orthogonal groups $G_{l}^{\text {ad }}$ in order to apply [Springer and Steinberg 1970]. As in Section 5.3, consider the centraliser $C_{\ell} \subset G_{A / \mathbb{Q}_{\ell}}$ of $\left(N_{\ell}^{\prime}, w_{\ell}\right)$ and note that $\rho_{\ell}^{\prime}\left(\Phi_{v}\right) \in \widetilde{C}_{\ell}\left(\mathbb{Q}_{\ell}\right)$, where $\widetilde{C}_{\ell}$ is the subgroup of $G_{A / \mathbb{Q}_{\ell}}$ generated by $C_{\ell}$ and the image of $w_{\ell}$. For any fixed embedding $\iota: L \rightarrow \overline{\mathbb{Q}}$, let $G_{\iota}$ be the image of $G_{A} / \overline{\mathbb{Q}}_{\ell}$ in $\mathrm{GL}\left(V_{\iota}\right)$. The centraliser $C_{\ell, \iota} \subset G_{\iota / \overline{\mathbb{Q}}_{\ell}}$ of $\left(N_{\ell, l}^{\prime}, w_{\ell, \iota}\right)$ can be described by projecting it to the adjoint group.

The action of $G_{\iota}$ on itself by conjugation factors through the adjoint group $G_{\iota}^{\text {ad }} \cong \mathrm{SO}_{2 n+1}$. Recall that $W_{\iota}$ is the orthogonal representation of this group. In view of [ibid., IV §2] and the dimension count carried out in Section 6.1, the centraliser $C_{\ell, \iota}^{\mathrm{b}} \subset G_{\iota / \overline{\mathbb{Q}}_{\ell}}^{\mathrm{ad}}$ of the pair $\left(N_{\ell, \iota}, w_{\ell, \iota}^{\mathrm{ad}}\right)$ satisfies

$$
C_{\ell, \iota}^{b} \cong \mathrm{SL}_{2 / \overline{\mathbb{Q}}_{\ell}} \times \mathrm{SO}_{2 n-3 / \overline{\mathbb{Q}}_{\ell}} .
$$

Consider the tori $T_{\iota} \subset \widetilde{T}_{\iota}$ defined in Section 6.1. Up to conjugation, we can assume that $w_{\ell, \iota}$ factors through $T_{\iota}$ and that its lift $\tilde{w}_{\ell, \iota}$ along $\mathbb{G}_{m}^{n+1} \rightarrow \widetilde{T}_{\iota}$ is given by

$$
\tilde{w}_{\ell, \iota}: \mathbb{G}_{m} \rightarrow \mathbb{G}_{m}^{n+1}, \quad t \mapsto\left(1, t^{1 / 2}, t^{1 / 2}, 1, \ldots, 1\right) .
$$

The image $\Delta^{\prime \prime} \subset T_{\iota} \subset \widetilde{T}_{\iota}$ of the map $t \mapsto\left(1, t^{1 / 2}, t^{-1 / 2}, 1, \ldots, 1\right)$ then projects to a maximal torus of the factor $\mathrm{SL}_{2}$ of $C_{\ell, l}^{b} \subset G_{\iota / \bar{Q}_{\ell}}^{\text {ad }}$. The product $\Delta^{\prime}=\mathbb{G}_{m}^{n-2}$ of the last $n-2$ factors projects to a maximal torus of the factor $\mathrm{SO}_{2 n-3}$.

The group $C_{\ell, \iota} \subset G_{\iota}$ is the inverse image of $C_{\ell, l}^{b}$. Its derived group therefore admits an isogeny

$$
C_{\ell, \iota}^{\prime \prime} \times C_{\ell, \iota}^{\prime}=\mathrm{SL}_{2 / \overline{\mathbb{Q}}_{\ell}} \times C_{\ell, \iota}^{\prime} \rightarrow C_{\ell, \iota}^{\mathrm{der}},
$$


where $C_{\ell, \ell}^{\prime}$ is a spin group of type $B_{n-2}$.

The map from $\Delta^{\prime \prime}$ to $G_{l}^{\text {der }}$ factors through $C_{\ell, l}^{\prime \prime} \times 1=\mathrm{SL}_{2}$ in the product above and the image of $\Delta^{\prime \prime}$ in $C_{\ell, \iota}^{\prime \prime}$ is a maximal torus. Similarly, $\Delta^{\prime}$ maps through $1 \times C_{\iota}^{\prime}$ and defines a maximal torus in $C_{\iota}^{\prime}$. Recall from Section 6.1 that $V_{\iota}$ is a multiple of the spin representation $V_{\iota}^{\text {irr }}$ of $G_{\iota}$. Considering the characters occurring in the representation of $\Delta^{\prime \prime} \times \Delta^{\prime}$ on $V_{l}^{\text {irr }}$, one concludes that, as a representation of $\mathrm{SL}_{2} / \overline{\mathbb{Q}}_{\ell} \times C_{\iota}^{\prime}$, the space $V_{\iota}^{\text {irr }}$ is a tensor product $V_{\iota}^{\prime \prime} \otimes V_{\iota}^{\prime}$. Here $V_{\iota}^{\prime \prime}$ is a multiple of the direct sum of the standard representation and two copies of the trivial representation of $\mathrm{SL}_{2}$ and $V_{\iota}^{\prime}$ is the spin representation of $C_{\iota}^{\prime}$. As representations of $C_{\ell, \iota}$, the $t$ and $t^{-1}$-eigenspaces $V_{\ell, \iota}^{0}$ and $V_{\ell, \iota}^{-2} \subset V_{\ell, \iota}$ of $w_{\ell, \iota}$ are both isomorphic to a multiple of $V_{\iota}^{\prime}$, so the representation of $C_{\ell, \iota}^{\prime \prime} \times C_{\ell, \iota}^{\prime}$ on $V_{\ell, \iota}^{0}$ identifies $C_{\ell, \iota}^{\prime}$ with its image in $\operatorname{GL}\left(V_{\ell, l}^{0}\right)$.

These observations imply that the isogeny above is in fact an isomorphism $C_{\ell, \iota}^{\prime \prime} \times C_{\ell, \iota}^{\prime}=\mathrm{SL}_{2 / \overline{\mathbb{Q}}_{\ell}} \times C_{\ell, \iota}^{\prime} \cong C_{\ell, \iota}^{\mathrm{der}}$ and hence

$$
C_{\ell / \overline{\mathbb{Q}}_{\ell}}^{\mathrm{der}} \cong \prod_{\iota: L \rightarrow \overline{\mathbb{Q}}_{\ell}}\left(C_{\ell, \ell}^{\prime \prime} \times C_{\ell, \ell}^{\prime}\right) \text {. }
$$

It also follows that $C_{\ell}^{\text {der }}$ itself decomposes as a product $C_{\ell}^{\prime \prime} \times C_{\ell}^{\prime}$ of algebraic groups over $\mathbb{Q}_{\ell}$. The group $C_{\ell}$ is generated by $C_{\ell}^{\text {der }}$ and the centre of $G_{A / \mathbb{Q}_{\ell}}$ and $\widetilde{C}_{\ell}$ is generated by $C_{\ell}$ and the image of $w_{\ell}$. We will show that $\rho_{\ell}^{\prime}\left(\Phi_{v}\right)$ lies in $\widetilde{C}_{\ell}^{\prime \prime} \subset \widetilde{C}_{\ell}$, the subgroup generated by $C_{\ell}^{\prime \prime}$ and the centre of $\widetilde{C}_{\ell}$.

Indeed, as in Section 5.4, we consider the action of $\rho_{\ell}^{\prime}\left(\Phi_{v}\right)$ on the different $w_{\ell}$-eigenspaces in $V_{\ell}$. It was pointed out in the beginning of the proof of Proposition 5.7 that, since $\rho_{\ell}^{\prime}\left(\Phi_{v}\right)$ is weakly neat, it acts on $V_{\ell}^{0}$ as multiplication by $\varepsilon= \pm 1$ and on $V_{\ell}^{-2}$ as multiplication by $\varepsilon q$. This means that $\rho_{\ell}^{\prime}\left(\Phi_{v}\right) \in \widetilde{C}_{\ell}^{\prime \prime}\left(\mathbb{Q}_{\ell}\right)$, as claimed.

The group $C_{\ell, \imath}^{\prime \prime}$, which is isomorphic to $\mathrm{SL}_{2}$, acts trivially on $V_{\ell, \iota}^{0}$ and on $V_{\ell, l}^{-2}$ and $V_{\ell, l}^{-1}$ is a multiple of the standard representation. The centre of $C_{\ell}$ acts on each $V_{\iota}$ through a fixed character. Through $w_{\ell}$, the group $\mathbb{G}_{m}$ acts on $V_{\ell}^{-2}$, on $V_{\ell}^{-1}$ and on $V_{\ell}^{0}$ as multiplication by $t^{-1}$, by 1 and by $t$, respectively. For the group $\widetilde{C}_{\ell}^{\prime \prime}$ defined above, this discussion implies that the map

$$
\widetilde{C}_{\ell}^{\prime \prime} \rightarrow \operatorname{GL}\left(V_{\ell}^{-2}\right) \times \operatorname{GL}\left(V_{\ell}^{-1}\right) \times \operatorname{GL}\left(V_{\ell}^{0}\right)
$$

is injective. As in the proof of Lemma 5.5, the image of $\rho_{\ell}^{\prime}\left(\Phi_{v}\right)$ under the map $P_{L}^{\prime}$, defined in $(5.4 *)$ by taking the $L$-linear characteristic polynomial on $V_{\ell}^{-1}$, lies in $\mathbb{A}_{(L)}^{2 g}(\mathbb{Q})$ and is independent of $\ell$. We already know that the images of $\rho_{\ell}^{\prime}\left(\Phi_{v}\right)$ in $\operatorname{GL}\left(V_{\ell}^{-2}\right)$ and in $\operatorname{GL}\left(V_{\ell}^{0}\right)$ are rational scalars, independent of $\ell$. In the case of an abelian variety of type $B_{n}$, the main theorem now follows using a variant of Lemma 5.8, again using [Noot 2009, 2.5, 2.6]. Note that, as before, the statement of [ibid., 2.6] is valid for any $\Omega$, instead of just $\mathbb{Q}$, because the group $\widetilde{C}_{\ell}^{\prime \prime}$ is of type $A_{1}$. 
6.3. Abelian varieties of type $D_{n}^{\mathbb{R}}$. The case where the abelian variety $A$ is strictly tractable with Mumford-Tate group of type $D_{n}^{\mathbb{R}}$ can be treated by analogous arguments. We will just indicate the points where the discussion of Sections 6.1 and 6.2 needs to be modified.

First of all, the quotient of $G_{A}$ one has to consider in order apply [Springer and Steinberg 1970] is not the adjoint group, but the intermediate quotient of $G_{A / \overline{\mathbb{Q}}_{\ell}}$ for which the simple factors $G_{\imath}^{b}$ are groups of the form $\mathrm{SO}_{2 n}$. Also, $A$ is not necessarily simple in this case. If it is not, then the endomorphism algebra $L$ is of the form $L=L^{\prime} \times L^{\prime}$, where $L^{\prime}$ is a number field; see Lemma 4.4.

We now follow the proof of [Noot 2009, Théorème 2.4] for this type. For each $\iota: L \rightarrow \overline{\mathbb{Q}}$, let $V_{\iota}$ be the direct factor of $V \otimes \overline{\mathbb{Q}}$ on which $L$ acts through $\iota$. The group $G_{A / \bar{Q}}^{\text {der }}$ acts on $V_{\iota}$ through a single direct factor $G_{\iota}^{\text {der }}$, but for $n \geq 4$ this factor does not act faithfully on $V_{\iota}$. In fact $V_{\iota}$ is a multiple of a semispin representation $V_{\iota}^{\text {irr }}$ of $G_{\iota}$, with highest weight $\varpi_{n-1}$ say. For $\iota=\iota^{+}$, there is a $\iota^{-}: L \rightarrow \overline{\mathbb{Q}}$ such that $V_{\iota^{-}}$is a multiple of the other semispin representation $V_{\iota^{-}}^{\text {irr }}$ of $G_{l}$, with highest weight $\varpi_{n}$. The representation of $G_{A / \overline{\mathbb{Q}}}$ on $V_{\iota^{+}} \oplus V_{l^{-}}$restricts to a faithful representation of $G_{\iota}^{\mathrm{der}}$. We redefine $G_{\iota}$ as the image of $G_{A / \bar{Q}}^{\mathrm{der}}$ in $\operatorname{GL}\left(V_{\iota^{+}}\right) \times \operatorname{GL}\left(V_{\iota^{-}}\right)$.

If $L$ is a field, then it is a CM field and the involution $\iota^{+} \mapsto \iota^{-}$on the set of maps $L \rightarrow \overline{\mathbb{Q}}$ defined by this construction is given by the composition with the complex conjugation on $L$. If $L=L^{\prime} \times L^{\prime}$ is a product of two fields, then $\iota^{-}$is the composite of $\iota^{+}$with the involution exchanging the factors. Using the spin representation $V_{l^{+}}^{\text {irr }} \oplus V_{l^{-}}^{\text {irr }}$ instead of $V_{\iota}^{\text {irr }}$, the arguments of Section 6.1 and hence the proof of Proposition 5.2 carry over to this case.

Where the discussion of Section 6.2 is concerned, the analogue of (6.2*) states that the centraliser $C_{\ell, \iota}^{\mathrm{b}}$ of $\left(N_{\ell, \iota}, w_{\ell, \iota}^{\mathrm{ad}}\right)$ in $G_{\iota}^{\mathrm{b}}$ is given by

$$
C_{\ell, \iota}^{b} \cong \mathrm{SL}_{2, \overline{\mathbb{Q}}_{\ell}} \times \mathrm{SO}_{2 n-4} \text {. }
$$

Once again, $C_{\ell, \iota} \subset G_{\iota}$ is the inverse image of $C_{\ell, \iota}^{b}$ and there is an isogeny

$$
C_{\ell, l}^{\prime \prime} \times C_{\ell, \iota}^{\prime} \cong \mathrm{SL}_{2 / \mathbb{Q}_{\ell}} \times C_{\ell, \iota}^{\prime} \rightarrow C_{\ell, \iota}^{\mathrm{der}} \text {. }
$$

Here the group $C_{\ell, l}^{\prime}$ is a spin group of type $D_{n-2}$. Similarly to the previous case, one shows that $V_{\iota^{+}}^{\text {irr }} \oplus V_{\iota^{-}}^{\text {irr }}$ is of the form $V_{\iota}^{\prime \prime} \otimes V_{\iota}^{\prime}$, where $V_{\iota}^{\prime \prime}$ is a multiple of the direct sum of the standard representation and two copies of the trivial representation of $\mathrm{SL}_{2}$ and $V_{\iota}^{\prime}$ is the spin representation of $C_{\iota}^{\prime}$. We prove once again that

$$
C_{\ell, \iota}^{\mathrm{der}}=C_{\ell, \iota}^{\prime \prime} \times C_{\ell, \iota}^{\prime}
$$

and we define $\widetilde{C}_{\ell}$ and $\widetilde{C}_{\ell}^{\prime \prime}$ as before. As in Section 6.2 one has $\rho_{\ell}^{\prime}\left(\Phi_{v}\right) \in \widetilde{C}_{\ell}^{\prime \prime}\left(\mathbb{Q}_{\ell}\right)$. For each pair $\iota^{+}, \iota^{-}$as above, the image of $C_{\ell}^{\prime \prime}$ in $C_{\ell, l^{+}}^{\prime \prime} \times C_{\ell, \iota^{-}}^{\prime \prime}$ is the graph of an isomorphism. Each $C_{\ell}^{\prime \prime}$ is again a group of type $A_{1}$, acting on the 1-eigenspace 
$V_{\iota^{+}}^{-1} \oplus V_{\iota^{-}}^{-1}$ for $w_{\ell}$ as a multiple of the standard representation. The argument can be completed as in Section 6.2.

\section{The proof of the main theorem, Theorem 3.6}

Proposition 7.1. Assume that we are in the situation of Theorem 3.6 and that the variety $A$ is tractable. Then there is a finite extension $F^{\prime}$ of $F$ such that Theorem 3.6 holds for $A_{/ F^{\prime}}$.

Proof. The Proposition 5.7 and the results of Section 6 prove the proposition in the case where $A$ is strictly tractable.

If $A$ is tractable then there exists a finite extension $F^{\prime} \supset F$, strictly tractable abelian varieties $A_{1}, \ldots A_{m} / F^{\prime}$ and an isogeny $A_{/ F^{\prime}} \sim \prod_{i=1}^{m} A_{i}$ such that the inclusion $f: G_{A} \rightarrow \prod_{i=1}^{m} G_{A_{i}}$ induces an isomorphism $G_{A}^{\mathrm{der}} \cong \prod_{i=1}^{m} G_{A_{i}}^{\mathrm{der}}$. In that case there are isomorphisms

$$
\mathfrak{g}_{A}^{\mathrm{ss}} \cong \bigoplus_{i=1}^{m} \mathfrak{g}_{A_{i}}^{\mathrm{ss}} \quad \text { and } \quad G_{A}^{\natural} \cong \prod_{i=1}^{m} G_{A_{i}}^{\natural} .
$$

For the induced map

$$
f_{\ell}: G_{A}\left(\mathbb{Q}_{\ell}\right) \rightarrow \prod_{i=1}^{m} G_{A_{i}}\left(\mathbb{Q}_{\ell}\right),
$$

one has $f_{\ell} \circ \rho_{A, \ell}=\left(\rho_{A_{i}, \ell}\right)_{i=1, \ldots, m}$, so the tangent map to $f_{\ell}$ sends the monodromy operator $N_{\ell}^{\prime} \in \mathfrak{g}_{A}^{\mathrm{ss}} \otimes \mathbb{Q}_{\ell}$ to the $m$-tuple in $\bigoplus_{i=1}^{m} \mathfrak{g}_{A_{i}}^{\mathrm{ss}} \otimes \mathbb{Q}_{\ell}$ of the monodromy operators associated to the $A_{i}$. This obviously implies that $f_{\ell} \circ \rho_{A, \ell}^{\prime}=\left(\rho_{A_{i}, \ell}^{\prime}\right)_{i=1, \ldots, m}$. The statement for $A_{/ F^{\prime}}$ therefore results immediately from the corresponding statements for the $A_{i}$.

7.2. Preliminaries to the proof of Theorem 3.6. We use the method of the proof of [Noot 2009, Théorème 1.8] in Section 3 of that paper. After fixing the notation we will indicate an omission, pointed out by Abhijit Laskar, in [Noot 2009] and explain how to complete the argument.

In what follows, the notation and the hypotheses of Section 3.1 and of Theorem 3.6 are in force, so $A$ has semistable reduction at $v$ and the image $\rho_{\ell}\left(\Phi_{v}\right)$ of the arithmetic Frobenius is weakly neat. However, since the argument involves auxiliary abelian varieties, we write $\left(\rho_{A, \ell}^{\prime}, N_{A, \ell}^{\prime}\right)$ for the representation of ${ }^{\prime} W_{v}$ associated to $A_{/ F_{v}}$.

By [Noot 2006, $\S 2$ and Corollary 3.2] of that paper, there is a tractable abelian variety $B_{/ \bar{F}}$ such that $B / \mathbb{C}$ provides a weak Mumford-Tate lift of $A / \mathbb{C}$. Following [Noot 2009, §3], this implies that there exists an abelian variety of CM-type $C_{/ \bar{F}}$ such that $A_{/ \bar{F}}$ belongs to the category of absolute Hodge motives generated by $B_{/ \bar{F}}$ and $C_{/ \bar{F}}$. This fact determines a morphism of Mumford-Tate groups $\pi: G_{B \times C} \rightarrow G_{A}$ 
but, contrary to what is stated in [ibid., §3], not a morphism $G_{B} \times G_{C} \rightarrow G_{A}$. In fact, $G_{B \times C}$ is a closed subgroup of the product $G_{B} \times G_{C}$ and the inclusion identifies the derived groups. This means that the diagram considered in the proof of [ibid., Théorème 1.8] has to be replaced by the diagram

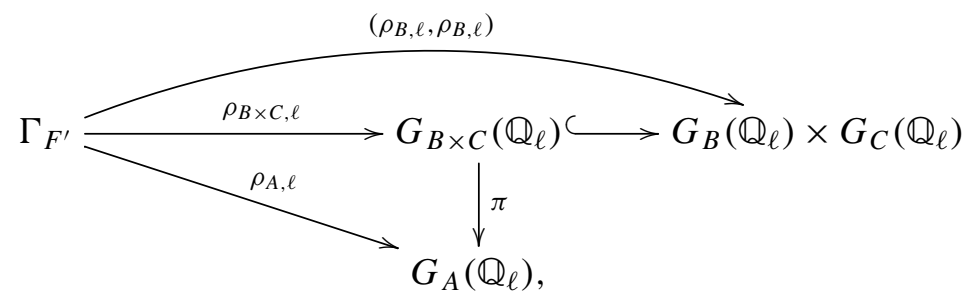

which commutes for a sufficiently large finite extension $F^{\prime}$ of $F$. None of the above depends on $v$ but this will not play any role in what follows.

7.3. Addendum to the proof of [Noot 2009, Théorème 1.8]. Recall that the statement of the theorem in question is essentially the special case of the main theorem of this paper where $A$ has good reduction. In [Noot 2009] it is formulated in terms of the variety of geometric conjugacy classes of the Mumford-Tate group. We have to prove that there exists a conjugacy class $\mathrm{Cl}_{A} \mathrm{Fr}_{v} \in \operatorname{Conj}^{\prime}\left(G_{A}\right)(\mathbb{Q})$ containing the image of $\rho_{A, \ell}\left(\Phi_{v}^{-1}\right)$ of any $\ell$ with $v(\ell)=0$. Here $\operatorname{Conj}^{\prime}\left(G_{A}\right) / \overline{\mathbb{Q}}$ is the quotient of $G_{A / \overline{\mathbb{Q}}}$ by the adjoint action of $G_{A}^{\natural \text { ad }}$. We refer to [ibid., 1.5] for the construction of a natural model $\operatorname{Conj}^{\prime}\left(G_{A}\right)$ over $\mathbb{Q}$. Assume for the moment that [ibid., Theorem 1.8] holds for $B \times C / F^{\prime}$, where $F^{\prime}$ is a sufficiently big finite extension of $F$ and $v^{\prime}$ an extension of the valuation $v$ to $F^{\prime}$. We then obtain a conjugacy class $\mathrm{Cl}_{B \times C} \mathrm{Fr}_{v^{\prime}} \in \operatorname{Conj}^{\prime}\left(G_{B \times C}\right)(\mathbb{Q})$ and its image $\mathrm{Cl}_{A} \operatorname{Fr}_{v^{\prime}} \in \operatorname{Conj}^{\prime}\left(G_{A}\right)(\mathbb{Q})$ fulfils the statement [ibid., Theorem 1.8] for $A_{/ F^{\prime}}$. The proof of Theorem 1.8 there then applies and it follows that the theorem also holds for $A / F$.

It remains to construct $\mathrm{Cl}_{B \times C} \operatorname{Fr}_{v^{\prime}}$. As $G_{C}$ is a torus, Conj' $\left(G_{B \times C}\right) / \overline{\mathbb{Q}}$ and Conj' $^{\prime}\left(G_{B} \times G_{C}\right) / \overline{\mathbb{Q}}$ are the quotients of $G_{B \times C / \overline{\mathbb{Q}}}$ and of $G_{B / \overline{\mathbb{Q}}} \times G_{C / \overline{\mathbb{Q}}}$ for the adjoint action by the same group, denoted $\operatorname{Aut}^{\prime}\left(G_{B}\right)$ in [Noot 2009, 1.5] and $G_{B}^{\natural \text { ad }}$ in Section 3.3 of this paper. If $T_{B \times C} \subset T_{B} \times G_{C}$ denote maximal tori of $G_{B \times C}$ and of $G_{B} \times G_{C}$, then $\operatorname{Conj}^{\prime}\left(G_{B \times C}\right)$ and $\operatorname{Conj}^{\prime}\left(G_{B} \times G_{C}\right)$ are also quotients of these tori by the finite group $\widetilde{W}$ of [ibid., 1.6]. The group $\widetilde{W}$ is an extension of a finite group of outer automorphisms by the Weyl group of $G_{B}^{\text {der }}$. We claim that the closed immersion $T_{B \times C} \subset T_{B} \times G_{C}$ induces a closed immersion on the quotients for the $\widetilde{W}$-action.

To justify the claim, assume that $R \rightarrow S$ is a surjective morphism of $\mathbb{Q}$-algebras with $\widetilde{W}$ action. Let $b \in S^{\widetilde{W}}$ and assume that $a \in R$ maps to $b$. The average of the elements of the $\widetilde{W}$-orbit of $a$ then is an element of $R^{\widetilde{W}}$ mapping to $b$. It follows that $R^{\widetilde{W}} \rightarrow S^{\widetilde{W}}$ is also surjective. 
As $B$ is tractable, Thórème 2.4 of [Noot 2009] provides a conjugacy class

$$
\left(\mathrm{Cl}_{B} \mathrm{Fr}_{v^{\prime}}, \mathrm{Cl}_{C} \mathrm{Fr}_{v^{\prime}}\right) \in \operatorname{Conj}^{\prime}\left(G_{B} \times G_{C}\right)(\mathbb{Q})
$$

containing $\left(\rho_{B, \ell}\left(\Phi_{v^{\prime}}^{-1}\right), \rho_{C, \ell}\left(\Phi_{v^{\prime}}^{-1}\right)\right)$ for any $\ell \neq p$. As $\left(\rho_{B, \ell}, \rho_{C, \ell}\right)$ factors through $G_{B \times C}$ for all $\ell$, it follows that $\left(\mathrm{Cl}_{B} \mathrm{Fr}_{v^{\prime}}, \mathrm{Cl}_{C} \mathrm{Fr}_{v^{\prime}}\right) \in \mathrm{Conj}^{\prime}\left(G_{B \times C}\right)(\mathbb{Q})$. It is obviously the class $\mathrm{Cl}_{B \times C} \mathrm{Fr}_{v^{\prime}}$ we had to construct.

Proof of Theorem 3.6. We take up the thread of the proof of Theorem 3.6 by considering the diagram (7.2*). In this diagram, the map $G_{B \times C} \hookrightarrow G_{B} \times G_{C}$ induces an isomorphism on the derived groups and it follows that $G_{B \times C}^{\natural \text { ad }}=G_{B}^{\natural \text { ad }}$ and that both the subgroup $G_{B \times C / \overline{\mathbb{Q}}} \subset\left(G_{B} \times G_{C}\right) / \overline{\mathbb{Q}}$ and the Lie subalgebra $\mathfrak{g}_{B \times C} \otimes \overline{\mathbb{Q}} \subset\left(\mathfrak{g}_{B} \oplus \mathfrak{g}_{C}\right) \otimes \overline{\mathbb{Q}}$ are stable under the adjoint action of $G_{B}^{\natural \text { ad }}$. Taking $F^{\prime}$ big enough and fixing an extension $v^{\prime}$ of the valuation, we can assume, by Proposition 7.1, that the conclusion of the main theorem holds for $B$. By [Noot 2009, Corollaire 2.2] we can also assume that it is valid for $C$. This implies that the theorem is true for $(B \times C)_{/ F^{\prime}}$.

Consider the statement of Theorem 3.6 for the representation of ${ }^{\prime} W_{F_{v^{\prime}}}$ associated to $A_{F^{\prime}}$. The monodromy operators are unaffected by passing from $A$ to $A_{F^{\prime}}$, whereas $\Phi_{v}$, and hence the $\rho_{A, \ell}^{\prime}\left(\Phi_{v}\right)$, are replaced by their $f$-th powers, where $f$ is the residue degree of the extension $F_{v^{\prime}}^{\prime} / F_{v}$. This exponent is independent of $\ell$.

The variety $C$ has potentially good reduction at $v^{\prime}$, so for every prime number $\ell$, the monodromy operator $N_{A, \ell}^{\prime} \in \mathfrak{g}_{A} \otimes \mathbb{Q}_{\ell}$ is the image of

$$
\left(N_{B, \ell}^{\prime}, 0\right) \in \mathfrak{g}_{B \times C} \otimes \mathbb{Q}_{\ell} \subset\left(\mathfrak{g}_{B} \oplus \mathfrak{g}_{C}\right) \otimes \mathbb{Q}_{\ell}
$$

under the tangent map to $\pi$. Here $N_{B, \ell}^{\prime}$ is the monodromy operator associated to $B / F_{v}$. We have made use of the fact, expressed by the diagram $(7.2 *)$, that the product of the $\ell$-adic Galois representations associated to $B$ and $C$ factors through $G_{B \times C}\left(\mathbb{Q}_{\ell}\right)$.

Similarly,

$$
\rho_{A, \ell}^{\prime}\left(\Phi_{v^{\prime}}\right)=\pi\left(\rho_{B, \ell}^{\prime}\left(\Phi_{v^{\prime}}\right), \rho_{C, \ell}^{\prime}\left(\Phi_{v^{\prime}}\right)\right),
$$

which makes sense since $\left(\rho_{B, \ell}^{\prime}\left(\Phi_{v^{\prime}}\right), \rho_{C, \ell}^{\prime}\left(\Phi_{v^{\prime}}\right)\right) \in G_{B \times C}\left(\mathbb{Q}_{\ell}\right) \subset\left(G_{B} \times G_{C}\right)\left(\mathbb{Q}_{\ell}\right)$. As the theorem holds for $(B \times C)_{/ F^{\prime}}$, it follows that the theorem is true for $A_{/ F^{\prime}}$.

Now return to the original field $F$. Let $\Omega \supset \mathbb{Q}_{\ell}$ be an algebraically closed field and $\sigma \in \operatorname{Aut}(\Omega)$. By what we just proved, the images of $\left(N_{A, \ell}^{\prime}, \rho_{\ell}^{\prime}\left(\Phi_{v}^{f}\right)\right)$ and $\sigma\left(N_{A, \ell}^{\prime}, \rho_{\ell}^{\prime}\left(\Phi_{v}^{f}\right)\right)$ in $\mathfrak{g}_{A} \otimes \Omega \times G_{A}(\Omega)$ are conjugate by an element $g \in G_{A}^{\natural}(\Omega)$. Thus $N_{A, \ell}^{\prime}=\operatorname{Ad}(g)\left(\sigma\left(N_{A, \ell}^{\prime}\right)\right)$ and we will show that $\rho_{\ell}^{\prime}\left(\Phi_{v}\right)=g \sigma\left(\rho_{\ell}^{\prime}\left(\Phi_{v}\right)\right) g^{-1}$ as well. Indeed, applying [Raynaud 1994, 4.2] as in Section 3.8, we obtain a strict 1-motive $M^{\prime} / F_{v}$ and a system of $\Gamma_{F_{v}}$-equivariant isomorphisms $V_{\ell}\left(A_{/ F_{v}}\right) \cong V_{\ell}\left(M^{\prime}\right)$. By Proposition 1.8, the characteristic polynomials of $\rho_{\ell}^{\prime}\left(\Phi_{v}\right)$ and $\sigma\left(\rho_{\ell}^{\prime}\left(\Phi_{v}\right)\right)$ acting on $V_{\ell}(A)$ coincide. This common polynomial is also the characteristic polynomial of 
$g \sigma\left(\rho_{\ell}^{\prime}\left(\Phi_{v}\right)\right) g^{-1}$. As we already know that $\rho_{\ell}^{\prime}\left(\Phi_{v}^{f}\right)=g \sigma\left(\rho_{\ell}^{\prime}\left(\Phi_{v}^{f}\right)\right) g^{-1}$, the equality $\rho_{\ell}^{\prime}\left(\Phi_{v}\right)=g \sigma\left(\rho_{\ell}^{\prime}\left(\Phi_{v}\right)\right) g^{-1}$ follows from Lemma 7.4 below.

Similarly, let $\Omega$ be an algebraically closed field containing $\mathbb{Q}_{\ell}$ and $\mathbb{Q}_{\ell^{\prime}}$. We know that the images of the pairs $\left(N_{A, \ell}^{\prime}, \rho_{\ell}^{\prime}\left(\Phi_{v}^{f}\right)\right)$ and $\left(N_{A, \ell^{\prime}}^{\prime}, \rho_{\ell^{\prime}}^{\prime}\left(\Phi_{v}^{f}\right)\right)$ are conjugate by some $g \in G_{A}^{\natural}(\Omega)$. Again by Proposition 1.8, the characteristic polynomials of $\rho_{\ell}^{\prime}\left(\Phi_{v}\right)$ and $\left.\rho_{\ell^{\prime}}^{\prime}\left(\Phi_{v}\right)\right)$ coincide so Lemma 7.4 implies that $\left.\rho_{\ell}^{\prime}\left(\Phi_{v}\right)=\rho_{\ell^{\prime}}^{\prime}\left(\Phi_{v}\right)\right)$. This proves the theorem for $A$.

Lemma 7.4. Assume that $\Omega$ is an algebraically closed field, $d>0$ is an integer and that $x, y \in \mathrm{GL}_{d}(\Omega)$ are two semisimple and weakly neat elements. Assume that $x^{f}=y^{f}$ for some integer $f$ and that $x$ and $y$ have the same characteristic polynomial. Then $x=y$.

Proof. This is a variant of [Noot 2009, Proposition 3.2].

For any semisimple element $z \in \mathrm{GL}_{d}(\Omega)$, let $T_{z} \subset \mathrm{GL}_{d}$ be the torus acting by scalar multiplication on each eigenspace of $z$. Up to conjugation, $z$ is a point of the diagonal torus $\mathbb{G}_{m}^{d} \subset \mathrm{GL}_{d}$ and, writing $t_{1}, \ldots, t_{d}$ for the coordinates on $\mathbb{G}_{m}^{d}$ and $z=\left(z_{1}, \ldots, z_{d}\right)$, one then has

$$
T_{z}=\left\{\left(t_{1}, \ldots, t_{d}\right) \in \mathbb{G}_{m}^{d} \mid t_{i}=t_{j} \text { if } z_{i}=z_{j}\right\} .
$$

Note that for every positive integer $n$ one has $T_{z^{n}} \subset T_{z}$ and that this inclusion is an equality if $z$ is weakly neat.

With this notation we prove the lemma. As $x$ and $y$ are weakly neat and satisfy $x^{f}=y^{f}$, we get $T_{x}=T_{x}=T_{y f}=T_{y}$. This implies in particular that $y \in T_{x}(\Omega)$. We can assume that $x$ lies in the diagonal torus $\mathbb{G}_{m}^{d} \subset \mathrm{GL}_{d}$ and we write $x=\left(x_{1}, \ldots, x_{d}\right) \in \mathbb{G}_{m}^{d}(\Omega)$. The fact that $x$ and $y$ have the same characteristic polynomial implies that there is a permutation $\sigma \in \mathrm{S}_{d}$ of the factors of the product $\mathbb{G}_{m}^{d}$ such that $y=\sigma(x)$. We have $x^{f}=y^{f}=\sigma\left(x^{f}\right)$ and, considering the equations (7.4*) for $T_{x^{f}}$, it follows that $\left.\sigma\right|_{T_{x} f}=\mathrm{id}$. Since $T_{x^{f}}=T_{x}$ we conclude that $x=\sigma(x)=y$ as claimed.

\section{References}

[Deligne 1973] P. Deligne, "Les constantes des équations fonctionnelles des fonctions $L$ ", pp. 501597 in Modular functions of one variable, II (Antwerp, 1972), edited by P. Deligne and W. Kuyk, Lecture Notes in Math. 349, Springer, Berlin, 1973. MR 50 \#2128 Zbl 0271.14011

[Deligne 1974a] P. Deligne, "La conjecture de Weil, I", Inst. Hautes Études Sci. Publ. Math. 43 (1974), 273-307. MR 49 \#5013 Zbl 0287.14001

[Deligne 1974b] P. Deligne, “Théorie de Hodge, III", Inst. Hautes Études Sci. Publ. Math. 44 (1974), 5-77. MR 58 \#16653b Zbl 0237.14003

[Deligne 1979] P. Deligne, "Variétés de Shimura: Interprétation modulaire, et techniques de construction de modèles canoniques", pp. 247-289 in Automorphic forms, representations and L-functions 
(Corvallis, OR, 1977), vol. 2, edited by A. Borel and W. Casselman, Proc. Sympos. Pure Math. 23, Amer. Math. Soc., Providence, R.I., 1979. MR 81i:10032 Zbl 0437.14012

[Deligne 1980] P. Deligne, "La conjecture de Weil, II”, Inst. Hautes Études Sci. Publ. Math. 52 (1980), 137-252. MR 83c:14017 Zbl 0456.14014

[Deligne 1982] P. Deligne, "Motifs et groupes de Taniyama”, pp. 261-279 in Hodge cycles, motives, and Shimura varieties, edited by P. Deligne et al., Lecture Notes in Mathematics 900, Springer, Berlin, 1982. Zbl 0499.14001

[Deligne and Milne 1982] P. Deligne and J. S. Milne, "Tannakian categories", pp. 101-228 in Hodge cycles, motives, and Shimura varieties, edited by P. Deligne et al., Lecture Notes in Mathematics 900, Springer, Berlin, 1982. Zbl 0477.14004

[Fontaine 1994] J.-M. Fontaine, "Représentations $l$-adiques potentiellement semi-stables", pp. 321347 in Périodes p-adiques (Bures-sur-Yvette, 1988), Astérisque 223, Société Mathématique de France, Paris, 1994. MR 95k:14031 Zbl 0873.14020

[Grothendieck et al. 1972] A. Grothendieck, M. Raynaud, and D. S. Rim, Groupes de monodromie en géométrie algébrique, I (SGA 7), Lecture Notes in Mathematics 288, Springer, Berlin, 1972. MR 50 \#7134

[Humphreys 1995] J. E. Humphreys, Conjugacy classes in semisimple algebraic groups, Mathematical Surveys and Monographs 43, Amer. Math. Society, Providence, RI, 1995. MR 97i:20057 Zbl 0834.20048

[Illusie 1994] L. Illusie, "Autour du théorème de monodromie locale", pp. 9-57 in Périodes padiques (Bures-sur-Yvette, 1988), Astérisque 223, Société Mathématique de France, Paris, 1994. MR 95k:14032 Zbl 0837.14013

[Ito 2004] T. Ito, "Weight-monodromy conjecture for certain threefolds in mixed characteristic", Int. Math. Res. Not. 2004:2 (2004), 69-87. MR 2005b:14033 Zbl 1090.14004

[Laskar 2011] A. Laskar, $\ell$-independence for a system of motivic representations, thesis, Université de Strasbourg, 2011, available at http://tinyurl.com/bpg6gen.

[Milne 1986] J. S. Milne, "Abelian varieties", pp. 103-150 in Arithmetic geometry (Storrs, CT, 1984), edited by G. Cornell and J. H. Silverman, Springer, New York, 1986. MR 861974 Zbl 0604.14028

[Mumford 1969] D. Mumford, "A note of Shimura's paper "Discontinuous groups and abelian varieties"”, Math. Ann. 181 (1969), 345-351. MR 40 \#1400 Zbl 0169.23301

[Mumford 1970] D. Mumford, Abelian varieties, Oxford University Press, 1970. MR 44 \#219 Zbl 0223.14022

[Noot 1995] R. Noot, "Classe de conjugaison du Frobenius des variétés abéliennes à réduction ordinaire”, Ann. Inst. Fourier (Grenoble) 45:5 (1995), 1239-1248. MR 96m:14064 Zbl 0834.14026

[Noot 2006] R. Noot, "Lifting systems of Galois representations associated to abelian varieties", $J$. Ramanujan Math. Soc. 21:4 (2006), 299-342. MR 2008a:11067 Zbl 1174.11050

[Noot 2009] R. Noot, "Classe de conjugaison du Frobenius d'une variété abélienne sur un corps de nombres", J. Lond. Math. Soc. (2) 79:1 (2009), 53-71. MR 2010e:14041 Zbl 1177.14084

[Ochiai 1999] T. Ochiai, "l-independence of the trace of monodromy", Math. Ann. 315:2 (1999), 321-340. MR 2000i:14024 Zbl 0980.14014

[Rapoport and Zink 1982] M. Rapoport and T. Zink, "Über die lokale Zetafunktion von Shimuravarietäten. Monodromiefiltration und verschwindende Zyklen in ungleicher Charakteristik", Invent. Math. 68:1 (1982), 21-101. MR 84i:14016 Zbl 0498.14010

[Raynaud 1994] M. Raynaud, "1-motifs et monodromie géométrique”, pp. 295-319 in Périodes p-adiques (Bures-sur-Yvette, 1988), Astérisque 223, Société Mathématique de France, Paris, 1994. MR 95h:14020 Zbl 0830.14001 
[Serre 1968] J.-P. Serre, Abelian l-adic representations and elliptic curves, W. A. Benjamin, New York, 1968. MR 41 \#8422 Zbl 0186.25701

[Serre 1994] J.-P. Serre, "Propriétés conjecturales des groupes de Galois motiviques et des représentations $l$-adiques", pp. 377-400 in Motives (Seattle, WA, 1991), edited by U. Jansen et al., Proc. Sympos. Pure Math. 55, Amer. Math. Soc., Providence, RI, 1994. MR 95m:11059 Zbl 0812.14002

[Serre 2000] J.-P. Serre, "Letters to Ribet, 1-1-1981 and 29-1-1981", pp. 1-20 in Euvres: Collected papers, vol. IV, Springer, Berlin, 2000.

[Serre and Tate 1968] J.-P. Serre and J. Tate, "Good reduction of abelian varieties", Ann. of Math. (2) 88 (1968), 492-517. MR 38 \#4488 Zbl 0172.46101

[Shimura and Taniyama 1961] G. Shimura and Y. Taniyama, Complex multiplication of abelian varieties and its applications to number theory, Publ. Math. Soc. Japan 6, Math. Soc. Japan, Tokyo, 1961. MR 23 \#A2419 Zbl 0112.03502

[Springer and Steinberg 1970] T. A. Springer and R. Steinberg, "Conjugacy classes", pp. 167-266 in Seminar on algebraic groups and related finite groups (Princeton, NJ, 1968/69), Lecture Notes in Mathematics 131, Springer, Berlin, 1970. MR 42 \#3091 Zbl 0249.20024

Communicated by Hélène Esnault

Received 2009-08-10 Revised 2012-01-17 Accepted 2012-03-25

noot@math.unistra.fr

Institut de Recherche Mathématique Avancée, UMR 7501, Université de Strasbourg and CNRS, 7 rue René Descartes, 67084 Strasbourg, France http://www-irma.u-strasbg.fr/ noot/ 


\section{Algebra \& Number Theory}

msp.org/ant

\section{EDITORS}

MANAGING EDITOR

Bjorn Poonen

Massachusetts Institute of Technology

Cambridge, USA

\author{
EDITORIAL BOARD CHAIR \\ David Eisenbud \\ University of California \\ Berkeley, USA
}

\section{BOARD OF EDITORS}

Georgia Benkart

Dave Benson

Richard E. Borcherds

John H. Coates

J-L. Colliot-Thélène

Brian D. Conrad

Hélène Esnault

Hubert Flenner

Edward Frenkel

Andrew Granville

Joseph Gubeladze

Ehud Hrushovski

Craig Huneke

Mikhail Kapranov

Yujiro Kawamata

János Kollár

Yuri Manin

Barry Mazur

Philippe Michel
University of Wisconsin, Madison, USA

University of Aberdeen, Scotland

University of California, Berkeley, USA

University of Cambridge, UK

CNRS, Université Paris-Sud, France

University of Michigan, USA

Freie Universität Berlin, Germany

Ruhr-Universität, Germany

University of California, Berkeley, USA

Université de Montréal, Canada

San Francisco State University, USA

Hebrew University, Israel

University of Virginia, USA

Yale University, USA

University of Tokyo, Japan

Princeton University, USA

Northwestern University, USA

Harvard University, USA

École Polytechnique Fédérale de Lausanne
Susan Montgomery

Shigefumi Mori

Raman Parimala

Jonathan Pila

Victor Reiner

Karl Rubin

Peter Sarnak

Joseph H. Silverman

Michael Singer

Vasudevan Srinivas

J. Toby Stafford

Bernd Sturmfels

Richard Taylor

Ravi Vakil

Michel van den Bergh

Marie-France Vignéras

Kei-Ichi Watanabe

Efim Zelmanov
University of Southern California, USA

RIMS, Kyoto University, Japan

Emory University, USA

University of Oxford, UK

University of Minnesota, USA

University of California, Irvine, USA

Princeton University, USA

Brown University, USA

North Carolina State University, USA

Tata Inst. of Fund. Research, India

University of Michigan, USA

University of California, Berkeley, USA

Harvard University, USA

Stanford University, USA

Hasselt University, Belgium

Université Paris VII, France

Nihon University, Japan

University of California, San Diego, USA

\section{PRODUCTION}

production@msp.org

Silvio Levy, Scientific Editor

See inside back cover or msp.org/ant for submission instructions.

The subscription price for 2013 is US \$200/year for the electronic version, and \$350/year ( $\$ 40$, if shipping outside the US) for print and electronic. Subscriptions, requests for back issues and changes of subscribers address should be sent to MSP.

Algebra \& Number Theory (ISSN 1944-7833 electronic, 1937-0652 printed) at Mathematical Sciences Publishers, 798 Evans Hall \#3840, c/o University of California, Berkeley, CA 94720-3840 is published continuously online. Periodical rate postage paid at Berkeley, CA 94704, and additional mailing offices.

ANT peer review and production are managed by EditFLOW ${ }^{\circledR}$ from Mathematical Sciences Publishers.

\section{PUBLISHED BY}

mathematical sciences publishers

nonprofit scientific publishing

http://msp.org/

(C) 2013 Mathematical Sciences Publishers 


\section{Algebra \& Number Theory}

$\begin{array}{lll}\text { Volume } 7 & \text { No. } 2 & 2013\end{array}$

The system of representations of the Weil-Deligne group associated to an abelian variety 243 RUTGER NOOT

Fourier-Jacobi coefficients of Eisenstein series on unitary groups

BEI ZHANG

The phase limit set of a variety

MoUnIR NisSE and FRANK SoTTILE

Base change behavior of the relative canonical sheaf related to higher dimensional moduli

Zsolt PATAKFALVI

Two ways to degenerate the Jacobian are the same

JESSE LEO KASS

Arithmetic motivic Poincaré series of toric varieties

Helena Cobo Pablos and Pedro Daniel González Pérez

Maximal ideals and representations of twisted forms of algebras

Michael LAU and ARTURo PiAnZOLA

Higher Chow groups of varieties with group action 